TRANSACTIONS OF THE

AMERICAN MATHEMATICAL SOCIETY

Volume 336, Number 1, March 1993

\title{
REYE CONSTRUCTIONS FOR NODAL ENRIQUES SURFACES
}

\author{
A. CONTE AND A. VERRA
}

\begin{abstract}
A classical Reye congruence $X$ is an Enriques surface of rational equivalence class $(3,7)$ in the grassmannian $G(1,3)$ of lines of $\mathbf{P}^{3} . X$ is the locus of lines of $\mathbf{P}^{3}$ which are included in two quadrics of $W=$ web of quadrics. A generalization to $G(1, t)$ is given (1) for each $t>2$ there exist Enriques surfaces $X$ of class $(t, 3 t-2)$ in $G(1, t),(2)$ the determinant of the dual of the universal bundle on $X$ is $\mathscr{O}_{X}\left(2 E+R+K_{X}\right)$, with $E=$ isolated elliptic curve, $R^{2}=-2, E \cdot R=t$, (3) $X$ parameterizes lines of $\mathbf{P}^{t}$ which are included in a codimension 2 subsystem of $W, W=$ linear system of quadrics of dimension $\left(\begin{array}{l}t \\ 2\end{array}\right)$. The paper includes a description of the variety of trisecant lines to a smooth Enriques surface of degree 10 in $\mathbf{P}^{5}$.
\end{abstract}

\section{INTRODUCTION AND PRELIMINARIES}

Let $X$ be an Enriques surface over $\mathbf{C}$,

$$
L=\operatorname{Num}(X)
$$

the group of its numerical equivalence classes. As it is well known

$$
L=\operatorname{Pic}(X) / \text { torsion } \cong \mathbf{Z}^{10}
$$

and, as a lattice, it is isomorphic to the orthogonal direct sum $E_{8} \oplus H$ (where $H$ is a hyperbolic plane and $E_{8}$ is defined as usual, cf. [10, p. 105]). In [10] F. Cossec and I. Dolgachev have studied $L$ in all details with the purpose of describing projective models of $X$; among them the so-called Fano models are of particular interest. Let us give first their construction; one has [10]:

(1.1) $L$ contains finitely many (modulo isometries) sets of isotropic vectors $\left\{e_{1}, \ldots, e_{10}\right\}$ such that

$$
e_{i} \cdot e_{j}=1-\delta_{i j}, \quad \frac{1}{3} \sum e_{i} \in L ;
$$

these sets always satisfy the following properties: let $|C|,\left|C^{\prime}\right|$ be the two linear systems of numerical class $\frac{1}{3} \sum e_{i}, C-C^{\prime}=K_{X}=$ canonical divisor of $X$, then

$$
C^{2}=10, \quad p_{a}(C)=6, \quad \operatorname{dim}|C|=5 .
$$

Assume $|C|$ is irreducible, then

Received by the editors July 2, 1990 and, in revised form, November 5, 1990.

1980 Mathematics Subject Classification (1985 Revision). Primary 14J28, 14 M15. 
(1.3) $\phi_{C}$ is a morphism of degree 1 and $X_{C}=\phi_{C}(X)$ is normal with at most rational double points;

(1.4) each divisor $C$ satisfying (1.2), (1.3) is obtained as in (1.1).

For an isotropic vector $e \in L$ we will say that $2 e$ corresponds to an elliptic pencil if there exist (exactly) two curves $E, E^{\prime}$ of class $e$ and such that $|2 E|=\left|2 E^{\prime}\right|$ is an elliptic pencil. Note that $E-E^{\prime} \sim K_{X}$ and $h^{0}\left(\mathscr{O}_{X}(E)\right)=$ $h^{0}\left(\mathscr{O}_{X}\left(E^{\prime}\right)\right)=1$; curves $E, E^{\prime}$ as above are said to be isolated elliptic curves on $X$. Let $C$ be as in (1.2), assume $C$ is very ample then $2 e_{i}$ corresponds to an elliptic pencil $\left|2 E_{i}\right|=\left|E_{i}^{\prime}\right|$. Moreover:

(1.5) $E_{i}, E_{i}^{\prime}$ are contained in $X_{C}$ as plane cubics; there is no other such curve in $X_{C}$.

The same holds for $C^{\prime}$. By definition $X_{C}\left(X_{C^{\prime}}\right)$ is a Fano model; $C\left(C^{\prime}\right)$ a Fano polarization. We recall that, on a general $X$, each Fano polarization is very ample and that $C$ very ample $\Leftrightarrow C^{\prime}$ very ample. To simply notations we will write $X$ instead of $X_{C}$ when no confusion arises.

Now let us describe the contents of this paper: our first purpose was to describe the variety $\operatorname{Tris}(X)$ of trisecant lines to a smooth Fano model $X$. This is done in $\S \S 2$ and 3: we show that, excluding one exceptional case, a line $L$ is trisecant to $X$ if and only if $L$ is trisecant to one of the twenty plane cubics in $X$. The exception is the following: Tris $(X)=\mathbf{P}^{3}$ blown up in twenty points; $X=X_{C^{\prime}}$, where $C^{\prime}=C+K_{X}$ is a Reye polarization.

We recall that a Reye polarization $C^{\prime}$ is a special Fano polarization, the special condition on it being

$$
X_{C^{\prime}} \subset G=\text { smooth quadric of } \mathbf{P}^{5} \text {. }
$$

If $C^{\prime}$ is a Reye polarization we will call $X_{C^{\prime}}$ a Reye congruence; the reason is that $G$ is the grassmannian of lines of $\mathbf{P}^{3}$ and that, traditionally, surfaces in $G$ (i.e. two dimensional families of lines of $\mathbf{P}^{3}$ ) were called congruence of lines.

The main modern result on Reye congruences is the following: every Reye congruence $X$ is a nodal Enriques surface and, conversely, on every nodal $X$ admitting a very ample Fano polarization there exists a Reye polarization too [8], [10, vol. II]. We recall that a nodal Enriques surface $X$ contains by definition a curve $R$ of arithmetic genus 0 and such that $h^{0}\left(\mathscr{O}_{R}\right)=1$. Following [10] we will say that $R$ is an indecomposable nodal cycle. By $[10$, p. 25] a curve $R$ in $X$ is an indecomposable nodal cycle iff it is a fundamental cycle of some rational double point.

Since nodal Enriques surfaces have no general moduli it follows that, for a general Enriques surface, there is no embedding in $G$ as a Fano model. Because of this remark, after the classification of $\operatorname{Tris}(X)$, we came on the following kinds of questions:

Let $L$ be a polarization on $X$ satisfying (1.3), $X_{L}=\phi_{L}(X), G_{r}$ the Plücker embedding of the grassmannian of lines of $\mathbf{P}^{r}$; when do we have

$$
X_{L} \subset \mathbf{P}^{N} \cap G_{r}, \quad r<<N ?
$$

$(N=\operatorname{dim}|L|)$, and what is the special feature of a projective model $X_{L}$ if $X$ is nodal?

To partially answer them we generalize the construction of Reye congruences to projective models of higher degree $L^{2}=4 t-2 \geq 10(\S \S 4$ and 5$)$ : 
Assume the set

$\mathscr{E}_{L}(t)=\left\{E \in \operatorname{Div}(X) / E=\right.$ isolated elliptic curve, $\left.E \cdot L=t, h^{1}\left(\mathscr{O}_{X}(L-2 E)\right)=0\right\}$ is not empty, then we show

$$
X_{L} \subset G_{t} \Leftrightarrow L \sim 2 E+R+K_{X} ;
$$

where $E \in \mathscr{E}_{L}(t), R=$ indecomposable nodal cycle, $E \cdot R=t$;

$$
\text { as a surface in } G_{t}, X \text { has rational equivalence class }(t, 3 t-2) \text {; }
$$

there exists a linear system $W$ of quadrics of $\mathbf{P}^{t}$ such that

$$
\operatorname{dim}(W)=\left(\begin{array}{l}
t \\
2
\end{array}\right) \quad \text { and } \quad X_{L}=\left\{l \in G_{t} \cap \mathbf{P}^{N} / \operatorname{codim}\left(W_{l}, W\right)=2\right\}
$$

with $W_{l}=\{Q \in W / l \subset Q\}, \mathbf{P}^{N}=$ linear span of $X$ in the Plücker space of $G_{t}$.

The case $t=3$ gives Reye congruences and their classical construction by a web of quadrics of $\mathbf{P}^{3}$. In view of this we define the projective model appearing in (1.6) as a Reye congruence of index $t$. Then, in $\S 6$, we show that Reye congruences of index $t$ exist for each $t \geq 3$.

Of course an embedding of $X$ in $G_{t}$ defines a rank 2 vector bundle on $X$ : the restriction to $X_{L}$ of the universal bundle of $G_{t}$. Let $\mathscr{Q}_{t}$ be the dual of such a bundle; by $(1.6),(1.7) c_{1}\left(\mathscr{Q}_{t}\right)^{2}=4 t-2, c_{2}\left(\mathscr{Q}_{t}\right)=t$; we can define $\mathscr{Q}_{t}$ as a Reye bundle of index $t$. This relates our results to those obtained by $\mathrm{I}$. Dolgachev and I. Reider in [11]: there they study the case $t=3$ and show that $Q_{3}$ is stable, (actually the unique stable rank 2 vector bundle on $X$ with Chern class $c_{1}^{2}=10, c_{2}=3$ ), and extremal i.e. without moduli. Moreover they are interested in the following problem: to find other examples of rank 2 vector bundles $\mathscr{E}$ on an Enriques surface which are stable and extremal.

In this case, computing the dimension of the moduli space, $\mathscr{E}$ must satisfy $c_{1}(\mathscr{E})^{2}=4 t-2, c_{2}(\mathscr{E})=t$; therefore our Reye bundle $\mathscr{Q}_{t}$ seems to be a natural candidate for further examples. During the completion of this paper we learned that stable-extremal rank 2 vector bundles on $X$ were described by Hoil Kim in his $\mathrm{Ph} . \mathrm{D}$. thesis [15]. In particular he produces examples $\mathscr{F}_{t}$ of them with $c_{2}\left(\mathscr{F}_{t}\right)=t$, any $t \geq 0$. We mention that, applying his results to our situation, it is possible to show that $\mathscr{Q}_{t}$ is stable-extremal too. Also, we have to mention that the description of $\operatorname{Tris}(X)$ for a Fano model $X$ is independently obtained in [11] as a consequence of the study of global sections of $\mathscr{Q}_{3}$.

Finally we wish to thank the referee for his help, especially in $\S 6$ where our previous degeneration arguments were considerably simplified by his suggestion of using Cremona transformations of $\mathbf{P}^{5}$ and generic nodal Enriques surfaces.

\section{Trisecants to Fano models}

Let $V \subset \mathbf{P}^{n}$ be a smooth projective variety, $L$ a line intersecting $V$; in the following we will say that $L$ is a trisecant line to $V$ if the scheme $V \cdot L$ is zero dimensional of length $\geq 3$; (hence, in particular, $L$ is not in $V$ ).

Consider a smooth hyperplane section $C=X \cap \mathbf{P}^{4}$ of a Fano model $X$; first we want to study the family $\operatorname{Tris}(C)$ of trisecant lines to $C$. By Berzolari formulae [12] the expected number of trisecant lines to a smooth, irreducible curve of genus $g$ and degree $d$ in $\mathbf{P}^{4}$ is $t=\frac{1}{6}(d-4)(d-3)(d-2)-g(d-4)$, 
which gives $t=20$ in our case. Note that $\mathscr{O}_{C}(1) \cong \omega_{C} \otimes \eta$, with $\eta=\mathscr{O}_{C}\left(K_{X}\right)=$ nontrivial order 2 element of $\operatorname{Pic}(C)$. On the other hand, for a general curve $D$ in $\mathbf{P}^{4}$ of degree 10 and genus $6, \mathscr{O}_{D}(1) \cong \omega_{D} \otimes \eta$, with $\eta=$ any degree zero line bundle.

So $C$ is a special element of its Hilbert scheme and, at least for this reason, we need to analyze more in detail $\operatorname{Tris}(C)$. For this consider any curve $C$ of genus 6 embedded in $\mathbf{P}^{4}$ by a very ample linear system $\omega_{C} \otimes \eta$ with $\eta=$ nontrivial order 2 element of $\operatorname{Pic}(C)$; let

$$
C(3)=3 \text {-symmetric product of } C \text {, }
$$$$
a: C(3) \rightarrow \mathrm{Pic}^{0}(C) \text { the Abel map, }
$$$$
W=a(C(3)) \text {, }
$$$$
W_{\eta}=\text { translation of } W \text { by } \eta \text {; then }
$$

(2.2) Proposition. Let $d \in C(3)$. There exists a trisecant line containing $d$ if and only if $h^{0}(\eta(d))=1$.

Proof. The condition $d$ is contained in a trisecant line is equivalent to $h^{0}\left(\omega_{C} \otimes \eta(-d)\right)=3$. Since $\eta$ is isomorphic to its dual $h^{0}\left(\omega_{C} \otimes \eta(-d)\right)=$ $h^{1}(\eta(d))$, (Serre duality). Finally, $h^{1}(\eta(d))=3 \Leftrightarrow h^{0}(\eta(d))=1$.

(2.3) Corollary. $d$ is contained in a trisecant line if and only if $a(d) \in W \cap W_{\eta}$.

Computing $W \cdot W_{\eta}$ we obtain by Poincaré formulae $W \cdot W_{\eta}=(\Theta / 3 !)^{2}=20$, $\left(\boldsymbol{\Theta}=\right.$ theta divisor in $\left.\mathrm{Pic}^{0}(C)\right)$. At this point we need a transversality condition for $W$ and $W_{\eta}$ in $\operatorname{Pic}^{0}(C)$ :

(2.4) Proposition. $W$ and $W_{\eta}$ are transversal at $a(d)$ if and only if $h^{0}(\eta(2 d))$ $=1$.

Proof. Since $h^{0}(\eta(d))=1$ there exists a unique $d^{\prime} \in C(3)$ such that $\eta \cong$ $\mathscr{O}_{C}\left(d^{\prime}-d\right)$. By $(2.2),(2.3) \quad a\left(d^{\prime}\right) \in W \cap W_{\eta}$ and $h^{0}\left(\eta\left(d^{\prime}\right)\right)=h^{0}\left(\mathscr{O}_{C}(d)\right)=$ 1. Moreover, since $\omega_{C} \otimes \eta$ is very $\operatorname{ample,} \operatorname{Supp}(d) \cap \operatorname{Supp}\left(d^{\prime}\right)=\varnothing[10$, $0.6]$. Writing the derivative of Abel map as in [1, 4.1] one obtains the standard identifications

$$
H^{0}\left(\omega_{C}\right) \cong T_{\operatorname{Pic}^{0}(C), a(d)}, \quad H^{0}\left(\omega_{C}(-d)\right) \cong T_{W, a(d)}
$$

since the derivative of the translation by $\eta$ is the identity, one has

$$
T_{W_{\eta}, a(d)}=T_{W, a\left(d^{\prime}\right)}=H^{0}\left(\omega_{C}\left(-d^{\prime}\right)\right) .
$$

Since $\operatorname{Supp}(d) \cap \operatorname{Supp}\left(d^{\prime}\right)=\varnothing$ we have $H^{0}\left(\omega_{C}\left(-d-d^{\prime}\right)\right)=H^{0}\left(\omega_{C}(-d)\right) \cap$ $H^{0}\left(\omega_{C}\left(-d^{\prime}\right)\right)$. Therefore, by Riemann-Roch and Serre duality,

$$
h^{0}\left(\omega_{C}\left(-d-d^{\prime}\right)\right)=0 \Leftrightarrow h^{1}(\eta(2 d))=0 \Leftrightarrow h^{0}(\eta(2 d))=1 \Leftrightarrow W, W_{\eta}
$$

are transversal at $a(d)$ (and also at $\left(a\left(d^{\prime}\right)\right)$.

Let us apply Proposition (2.4) to a smooth hyperplane section $C=\mathbf{P}^{4} \cap X$ of a smooth Fano model. Of course the 10 pairs $E_{i}, E_{i}^{\prime}(i=1 \cdots 10)$ of plane cubic curves define 10 pairs $L_{i}, L_{i}^{\prime}$ of trisecant lines to $C$ and the divisors

$$
d_{i}=E_{i} \cdot C=L_{i} \cdot C, \quad d_{i}^{\prime}=E_{i}^{\prime} \cdot C=L_{i}^{\prime} \cdot C
$$


on $C$. Note that $\mathscr{O}_{C}\left(d_{i}^{\prime}-d_{i}\right) \cong \mathscr{O}_{C}\left(K_{X}\right) \cong \eta$, where $\omega_{C} \otimes \eta=\mathscr{O}_{C}(1)$. Therefore we have

$$
\eta\left(2 d_{i}\right) \cong \mathscr{O}_{C}\left(E_{i}+E_{i}^{\prime}\right) \cong \eta\left(2 d_{i}^{\prime}\right)
$$

and the exact sequence

$$
0 \rightarrow \mathscr{O}_{X}\left(-C+E_{i}+E_{i}^{\prime}\right) \rightarrow \mathscr{O}_{X}\left(E_{i}+E_{i}^{\prime}\right) \rightarrow \eta\left(2 d_{i}\right) \rightarrow 0 .
$$

Since $h^{1}\left(\mathscr{O}_{X}\left(E_{i}+E_{i}^{\prime}\right)\right)=0$ this gives the exact sequence

$$
0 \rightarrow H^{0}\left(\mathscr{O}_{X}\left(E_{i}+E_{i}^{\prime}\right)\right) \rightarrow H^{0}\left(\eta\left(2 d_{i}\right)\right) \rightarrow H^{1}\left(\mathscr{O}_{X}\left(-C+E_{i}+E_{i}^{\prime}\right)\right) \rightarrow 0
$$

with $\operatorname{dim} H^{0}\left(\mathscr{O}_{X}\left(E_{i}+E_{i}^{\prime}\right)\right)=1$. Therefore we have shown

(2.10) Proposition. $W$ and $W_{\eta}$ are transversal at $a\left(d_{i}\right), a\left(d_{i}^{\prime}\right)$ if and only if $H^{1}\left(\mathscr{O}_{X}\left(-C+E_{i}+E_{i}^{\prime}\right)\right)=0$.

The main point is now the following known result:

(2.11) Proposition. Let $X$ be a smooth Fano model polarized by $C$, then $h^{1}\left(\mathscr{O}_{X}\left(-C+E_{i}+E_{i}^{\prime}\right)\right)=0 \quad \forall i=1 \cdots 10$ unless $C+K_{X}$ is a Reye polarization.

Proof. Cf. [8] and the next section.

(2.12) Theorem. Let $X$ be a smooth Fano model polarized by $C, \pi_{i}, \pi_{i}^{\prime}$ be the planes containing $E_{i}, E_{i}^{\prime} ; \pi_{i}^{*}, \pi_{i}^{\prime *}$ their dual planes. Assume $C+K_{X}$ is not a Reye polarization then

$$
\operatorname{Tris}(X)=\bigcup_{i=1}^{10}\left(\pi_{i}^{*} \cup \pi_{i}^{\prime *}\right) .
$$

Proof. Let $L \in \operatorname{Tris}(X)$, by definition $L$ is not in $X$ so that there exists a $\mathbf{P}^{4}$ transversal to $X$ and containing $L$. Let $C=X \cap \mathbf{P}^{4}, l$ a degree 3 divisor contained in the 0 -cycle $L \cdot C$. Then $a(l) \in W \cap W_{\eta} \subset \operatorname{Pic}^{0}(C)$. By $(2.4),(2.11)$ the scheme $W \cdot W_{\eta}$ contains the points $a\left(d_{i}\right), a\left(d_{i}^{\prime}\right),(i=$ $1 \cdots 10)$, as isolated components. Let $Z$ be possibly some different excess intersection component of $W \cdot W_{\eta}$. Let us show directly that $Z=0$ : consider in $C(3) \times \operatorname{Pic}^{0}(C)$ the incidence correspondence $I=\left\{(d, \xi) / h^{0}(\xi(d)) \geq 1\right\}$. The projection $p_{1}: I \rightarrow C(3)$ has fibre isomorphic to $W,(\forall d \in C(3))$. Hence $I$ is irreducible, $\operatorname{dim}(I)=6$. The projection $p_{2}: I \rightarrow \operatorname{Pic}^{0}(C)$ has fibre $W \cdot W_{\xi}$ over $\xi \quad\left(W_{\xi}=W\right.$ translated by $\left.\xi\right)$; moreover $p_{2}$ is surjective and $\operatorname{deg}\left(p_{2}\right)=$ $W^{2}=20$. Applying Stein factorization to $p_{i}$ it follows that the number of connected components of $p_{2}^{-1}(\xi)=W \cap W_{\xi}$ cannot exceed 20 . Hence $W \cdot W_{\eta}=$ $\sum\left(a\left(d_{i}\right)+a\left(d_{i}^{\prime}\right)\right), l=d_{i}$ or $d_{i}^{\prime}$ for some $i, L \in \pi_{i}^{*}$ or $\pi_{i}^{\prime *}$.

(2.13) Remark. Take a smooth Fano model $X$ satisfying the assumption of Theorem 2.12, then a general point $p$ of $X$ is not contained in a trisecant line to $X$. Hence projecting $X$ from $p$ one obtains a smooth Enriques surface $S \subset \mathbf{P}^{4}$ of degree 9 and sectional genus 6 which has been blown up in one point. The existence of such an $S$ was previously conjectured. A proof has been recently given in [13] by using a different method.

\section{TRISECANTS to adjoINTS to ReYE MODELS}

To complete the results of the previous section we need to describe $\operatorname{Tris}\left(X_{C}\right)$ in the case $C+K_{X}=$ very ample Reye polarization. With this purpose we 
give an explicit projective construction for $X_{C}$ which essentially comes from the results of $[8,10]$. Nevertheless we present it as a special case of the more general situation to be discussed in $\S 4$. Let

$$
V=\text { vector space, } \quad \operatorname{dim}(V)=t+1, \quad \mathbf{P}^{t}=\mathbf{P}(V) .
$$

Consider the Segre product

$$
\mathbf{P}^{t} \times \mathbf{P}^{t} \subset \mathbf{P}^{\otimes}=\mathbf{P}(V \otimes V)
$$

(i.e. the projectivized set of indecomposable vectors of $V \otimes V$ ) and the involution

$$
I_{t}: \mathbf{P}^{t} \times \mathbf{P}^{t} \rightarrow \mathbf{P}^{t} \times \mathbf{P}^{t}
$$

induced by the linear map $v_{1} \otimes v_{2} \rightarrow v_{2} \otimes v_{1}$. Let $Q^{+}, Q^{-}$be its eigenspaces, they are generated respectively by vectors $\left(v_{1} \otimes v_{2}+v_{2} \otimes v_{1}\right)$ and $\left(v_{1} \otimes v_{2}-\right.$ $\left.v_{2} \otimes v_{1}\right)$. Hence, as usual, we identify $Q^{+}, Q^{-}$to $\operatorname{Sym}^{2} V, \wedge^{2} V$ by the isomorphisms $\left(v_{1} \otimes v_{2}+v_{2} \otimes v_{1}\right) \rightarrow v_{1} v_{2}$ and $\left(v_{1} \otimes v_{2}-v_{2} \otimes v_{1}\right) \rightarrow v_{1} \wedge v_{2}$. Correspondingly we have in $\mathbf{P}^{\otimes}$ the projectivized eigenspaces

$$
\mathbf{P}^{+}=\mathbf{P}\left(\operatorname{Sym}^{2} V\right), \quad \mathbf{P}^{-}=\mathbf{P}\left(\bigwedge^{2} V\right)
$$

which are the set of fixed points of $I_{t}$. We are interested in the linear projections

$$
p_{+}: \mathbf{P}^{\otimes} \rightarrow \mathbf{P}^{+}, \quad p_{-}: \mathbf{P}^{\otimes} \rightarrow \mathbf{P}^{-}
$$

respectively of centers $\mathbf{P}^{-}, \mathbf{P}^{+}$; restricting these projections to $\mathbf{P}^{t} \times \mathbf{P}^{t}$ we get

$$
\mathbf{P}^{-} \stackrel{p_{-}}{\longleftarrow} \mathbf{P}^{t} \times \mathbf{P}^{t} \stackrel{p_{+}}{\rightarrow} \mathbf{P}^{+}
$$

with $p_{+}\left(v_{1} \otimes v_{2}\right)=v_{1} v_{2}, p_{-}\left(v_{1} \otimes v_{2}\right)=v_{1} \wedge v_{2}$. Therefore

$$
p_{-}\left(\mathbf{P}^{t} \times \mathbf{P}^{t}\right)=G_{t}, \quad p_{+}\left(\mathbf{P}^{t} \times \mathbf{P}^{t}\right)=\Sigma_{t}
$$

with $G_{t}=$ Plücker embedding of the grassmannian of lines of $\mathbf{P}^{t}, \Sigma_{t}=2$ symmetric product of $\mathbf{P}^{t}$. Since $p_{+}$is the quotient map $\mathbf{P}^{t} \times \mathbf{P}^{t} \rightarrow \mathbf{P}^{t} \times \mathbf{P}^{t} /\left\langle I_{t}\right\rangle$ it turns out that $\operatorname{Sing}\left(\Sigma_{t}\right)=p_{+}(\Delta)$, where $\Delta$ is the diagonal of $\mathbf{P}^{t} \times \mathbf{P}^{t}$; it is known that each point of $\operatorname{Sing}\left(\Sigma_{t}\right)$ occurs with multiplicity $2 t-2$. Finally $\operatorname{deg} \Sigma_{t}=\frac{1}{2}\left(\begin{array}{c}2 t \\ t\end{array}\right)$.

Consider the case $t=3$, then $\mathbf{P}^{-}=\mathbf{P}^{5}, \mathbf{P}^{+}=\mathbf{P}^{9}, \mathbf{P}^{\otimes}=\mathbf{P}^{15}$. Let $\Sigma=\Sigma_{3}$, $G=G_{3}$; fix any 5-dimensional projective space

$$
\Lambda \subset \mathbf{P}^{+}
$$

intersecting properly $\Sigma$ along the reduced, irreducible surface of degree 10

$$
X=\Lambda \cdot \Sigma \text {. }
$$

Assume $X$ is normal with at most rational double points, then

(3.10) Proposition. $X$ is a Fano model. Let $C$ be its polarization, then $C+K_{X}$ is a Reye polarization.

Proof. Since $X$ has no point of multiplicity $4, \operatorname{Sing}(\Sigma) \cap X=\varnothing$. Let $\tilde{X}=$ $p_{+}^{-1}(X)$, then $p_{+}: \widetilde{X} \rightarrow X$ is an étale double covering and $i=I_{t} / \widetilde{X}$ its associated fixed points free involution; moreover $\tilde{X}$ is normal with at most rational 
double points. Note that $\tilde{X}$ is a complete intersection in $\mathbf{P}^{3} \times \mathbf{P}^{3}$ of four hyperplane sections; by Bertini's theorem this implies that $\tilde{X}$ is connected and, since it is normal, irreducible. Finally, by adjunction formula, $\widetilde{X}$ is a $K 3$-surface. Since $i$ is fixed-point-free and $X=\widetilde{X} /\langle i\rangle, X$ is an Enriques surface and, by (1.4), a Fano model.

Now consider the other projection $p_{-}: \widetilde{X} \rightarrow \mathbf{P}^{-} ; p_{-}(\widetilde{X})$ is a Fano model of $X$ which is contained in $G$, hence a Reye congruence. Let $C^{\prime}$ be its polarization: clearly $C \nsim C^{\prime}$; on the other hand the pull back of $\mathscr{O}_{X}\left(C^{\prime}-C\right)$ to $\widetilde{X}$ is trivial. Since, as an étale double covering of $X, \widetilde{X}$ is defined by $\mathscr{O}_{X}\left(K_{X}\right)$, it follows $C-C^{\prime} \sim K_{X}$.

(3.11) Lemma. Let $X_{C}$ be a smooth Fano model;

$$
(u, v)=\left(h^{1}\left(\mathscr{O}_{X}\left(E_{i}+E_{i}^{\prime}-C\right)\right), h^{1}\left(\mathscr{O}_{X}\left(C^{\prime}-2 E_{i}\right)\right)\right)
$$

Then $(u, v)$ must be one of the pairs $(0,0),(1,0),(0,1)$.

Proof. Let for instance $u>0$; applying Riemann Roch it follows that $C^{\prime}-$ $E_{i}-E_{i}^{\prime} \sim R$ with $R$ effective, $p_{a}(R)=0$. Consider the exact sequence

$$
0 \rightarrow H^{0}\left(\mathscr{O}_{X}(-R)\right) \rightarrow H^{0}\left(\mathscr{O}_{X}\right) \rightarrow H^{0}\left(\mathscr{O}_{R}\right) \rightarrow H^{1}\left(\mathscr{O}_{X}(-R)\right) \rightarrow 0,
$$

by Serre duality $v=h^{1}\left(\mathscr{O}_{X}(-R)\right)$. Since $R$ is a curve of degree 4 in the smooth model $X_{C}$ it is easy to check that $v>0 \Leftrightarrow R$ is not connected. Assume $v>0$ then $R=A+B$ with $A, B$ effective, $A \cap B=\varnothing$. Since $E_{i} \cdot(A+B)=3$ we have $E_{i} \cdot A$ or $E_{i} \cdot B \leq 1$. Hence the linear system $\left|E_{i}+A+B\right|=\left|C^{\prime}-E_{i}^{\prime}\right|$ is not numerically 2 -connected. On the other hand, $\left|C^{\prime}-E_{i}^{\prime}\right|$ is base-point-free [14] hence numerically 2 -connected [10, 4.3.4, 4.4.1], contradiction. Therefore it must be $v=0$. Since $R$ is connected and $p_{a}(R)=0, h^{0}\left(\mathscr{O}_{X}(R)\right)=1$. Hence $h^{0}\left(\mathscr{O}_{X}(R)\right)=h^{0}\left(\mathscr{O}_{X}\left(C^{\prime}-E_{i}-E_{i}^{\prime}\right)\right)=h^{1}\left(\mathscr{O}_{X}\left(E_{i}+E_{i}^{\prime}-C\right)\right)=u=1$. A completely similar argument works if we assume $v>0$.

(3.12) Lemma. Assume $C^{\prime}=C+K_{X}$ is a very ample Reye polarization, then $h^{1}\left(\mathscr{O}_{X}\left(C^{\prime}-2 E_{i}\right)\right)=0, i=1 \cdots 10$.

Proof. Let $\pi_{i}, \pi_{i}^{\prime}$ be the supporting planes of the cubic curves $E_{i}, E_{i}^{\prime}$ in the smooth Reye model $X_{C^{\prime}}$; it is very well known that $\pi_{i} \cap \pi_{i}^{\prime} \neq \varnothing$. Hence $h^{0}\left(\mathscr{O}_{X}\left(C^{\prime}-E_{i}-E_{i}^{\prime}\right)\right)=h^{1}\left(\mathscr{O}_{X}\left(E_{i}+E_{i}^{\prime}-C\right)\right)>0$ and, by Lemma 3.11, $h^{1}\left(\mathscr{O}_{X}\left(C^{\prime}-2 E_{i}\right)\right)=0$.

(3.13) Proposition. Let $X$ be a smooth Fano model, then the following conditions are equivalent:

(1) $h^{1}\left(\mathscr{O}_{X}\left(E_{i}+E_{i}^{\prime}-C\right)\right)=1$ for some $i=1 \cdots 10$,

(2) $C \sim 2 E+R, E=$ isolated elliptic curve, $R=$ indecomposable nodal cycle, $E \cdot R=3$,

(3) $C^{\prime}=C+K_{X}$ is a Reye polarization,

(4) $h^{1}\left(\mathscr{O}_{X}\left(E_{i}+E_{i}^{\prime}-C\right)\right)=1$ for each $i=1 \cdots 10$,

(5) $X_{C}=\Lambda \cdot \Sigma$ as in (3.9).

Proof. Consider as in (4.11) $\mathscr{E}_{C}=\{E \in \operatorname{Div}(X) / E=$ isolated elliptic curve, $\left.E \cdot C=3, h^{1}\left(\mathscr{O}_{X}\left(C^{\prime}-2 E\right)\right)=0\right\}$. By Lemmas (3.11), (3.12) the cubic curves $E_{i}, E_{i}^{\prime}$ belong to $\mathscr{E}_{C}(3)$. Then the equivalence of (1), (2), (3), (4) is just the case $t=3$ of Theorem (4.12). Finally (3) $\Rightarrow(5)$ by Lemma 5.6 and (5) $\Rightarrow(3)$ by Proposition 3.10. 
(3.14) Proposition. Let $X$ be a smooth Fano model polarized by $C$. Assume $C+K_{X}$ is Reye then

(1) $\operatorname{Tris}(X)$ is the blowing up of $\mathbf{P}^{3}$ in 20 points;

(2) the union of trisecant lines to $X$ is a determinantal quartic hypersurface $\Delta=\left\{\operatorname{det}\left(d_{i j}\right)=0\right\}$, where $\left(d_{i j}\right)$ is a $4 \times 4$ symmetric matrix of linear forms.

Proof. By (3.11), $C+K_{X}=$ Reye polarization $\Leftrightarrow X=\Lambda \cdot \Sigma \subset \mathbf{P}\left(\operatorname{Sym}^{2} V\right)$ as in (3.9). $\mathbf{P}\left(\operatorname{Sym}^{2} V\right)$ is the parameter space for quadrics $Q$ in $\mathbf{P}\left(V^{*}\right)=\mathbf{P}^{3}$ and $\Sigma$ parametrizes rank 2 quadrics. Hence $\Lambda$ is a 5 -dimensional linear system of quadrics of $\mathbf{P}^{3 *}$ and

$$
X=\Lambda \cdot \Sigma=\{Q \in \Lambda / \operatorname{rank}(Q)=2\} .
$$

Let $p \in \mathbf{P}^{3 *}, \Lambda_{p}=\left\{Q \in \Lambda / p \in \operatorname{Sing}(Q), \operatorname{dim}\left(\Lambda_{p}\right)=d\right\}$. If $d=1$ then, counting properly multiplicities, $\Lambda_{p}$ contains three rank 2 quadrics and it is a trisecant line to $X$. In the same way $d=2 \Rightarrow \Lambda_{p} \cdot X=$ plane cubic: we know that there are exactly 20 points $o_{i}(i=1 \cdots 20)$ such that $d=2$. If $d=3$ then $\Lambda$ contains a rank 1 quadric, which is a point of multiplicity 4 for $\Lambda \cdot \Sigma=X$ : against our assumptions. Let $P$ be a 3-secant line to $X, \Delta$ the quartic hypersurface parametrizing singular quadrics of $\Lambda$. Since $\operatorname{Sing}(\Delta)=\Lambda \cdot \Sigma=X$ it follows that $P \subset \Delta$. Since, by definition $P$ is not in $X$, all the members of $P$ have rank 3 but for three of them having rank 2 . Looking at the projective classification of pencil of quadrics one can deduce that, in our case, $P$ has a (unique) singular base point $p$. This yields a morphism $f: \operatorname{Tris}(X) \rightarrow \mathbf{P}^{3}$ sending $P$ to $p . f$ blows up $\mathbf{P}^{3}$ in the points $o_{i}$. The union of trisecant lines to $X$ is the quartic hypersurface considered above. This completes the proof: for brevity we omitted some easy details.

\section{ENRIQUES SURFACES IN GRASSMANNIANS}

In this section we construct projective models $X$ of Enriques surfaces which are contained in the intersection of $G_{t}$ with a $(2 t-1)$-space: $X \subset \mathbf{P}^{2 t-1} \cap G_{t}$. The degree of $X$ in $\mathbf{P}^{2 t-1}$ is $4 t-2$, the rational equivalence class of $X$ in $G_{t}$ is $(t, 3 t-2)$. These models are the natural generalization of classical Reye congruences.

Let $\mathscr{H}_{t}, t \geq 3$, be the Hilbert scheme of normal Enriques surfaces of degree $4 t-2, \mathscr{R}_{t}$ the Hilbert scheme of normal Enriques surfaces in $G_{t}$ having rational equivalence class $(t, 3 t-2), f: \mathscr{R}_{t} \rightarrow \mathscr{H}_{t}$ the obvious morphism between these two Hilbert schemes. As a consequence of our generalization we would like to conjecture the following:

$$
f\left(\mathscr{R}_{t}\right) \text { is a closed codimension one subset of } \mathscr{H}_{t} \text { and its points }
$$
correspond to all nodal Enriques surfaces.

For Fano models $(t=3)$ this is generically true, [8,10]. Let $L$ be an irreducible polarization on $X$ such that

$$
L^{2}=4 t-2, \quad \phi_{L} \text { is a degree } 1 \text { morphism, } \quad X_{L} \text { is normal. }
$$

(4.3) Definition. We say that the pair $(X, L)$ fits in a Reye diagram of index $t$ if the following conditions are satisfied 
(1) there exists a commutative diagram

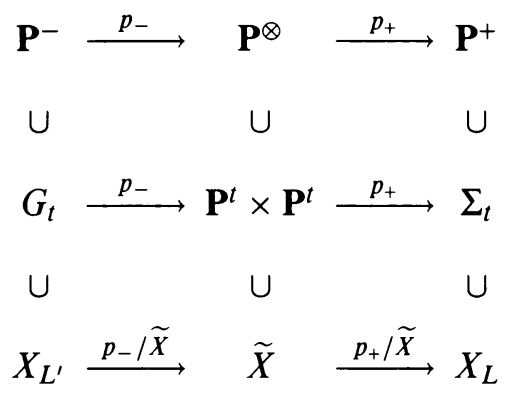

where $\tilde{X}$ is the $K 3$ cover of $X^{\prime}, L^{\prime}=K_{X}+L$ and for the top arrow we use the same notations of (3.1)-(3.6).

(2) The projections of $\widetilde{X}$ on the two factors of $\mathbf{P}^{t} \times \mathbf{P}^{t}$ are not contained in a hyperplane.

(4.5) Proposition. Assume $L=2 E+R$, with $E=$ isolated elliptic curve, $R=$ indecomposable nodal cycle, $E \cdot L=t$. Then the pair $(X, L)$ fits in a Reye diagram of index $t$.

Proof. $L^{2}=4 t-2$; let $\pi: \widetilde{X} \rightarrow X$ be the $K 3$ étale double cover of $X$, $i: \widetilde{X} \rightarrow \widetilde{X}$ the induced involution, $\widetilde{L}=\pi^{*} L$. Since $R$ is connected $\pi^{*} R$ splits

$$
\pi^{*} R=R_{1}+R_{2},
$$

$\pi / R_{j}: R_{j} \rightarrow R=$ isomorphism $(j=1,2)$. This yields two polarizations of degree $2 t-2$ on $\tilde{X}$ :

$$
L_{1}=R_{1}+\widetilde{E}, \quad L_{2}=R_{2}+\widetilde{E}
$$

with $|\widetilde{E}|=\left|\pi^{*} E\right|=$ elliptic pencil. Since $h^{1}\left(\mathscr{O}_{\widetilde{X}}\left(R_{j}\right)\right)=0$ the sequence

$$
0 \rightarrow H^{0}\left(\mathscr{O}_{\widetilde{X}}\left(R_{j}\right)\right) \rightarrow H^{0}\left(\mathscr{O}_{\widetilde{X}}\left(L_{j}\right)\right) \rightarrow H^{0}\left(\mathscr{O}_{\widetilde{E}}\left(L_{j}\right)\right) \rightarrow 0
$$

is exact and $\operatorname{dim}\left|L_{j}\right|=t$. Let $\phi_{j}: \widetilde{X} \rightarrow \mathbf{P}^{t}$ be the map defined by $\left|L_{j}\right|, X_{j}=$ $\phi_{j}(\widetilde{X})$ : by (4.6) and the very ampleness of $\mathscr{O}_{\widetilde{E}}\left(L_{j}\right), \phi_{j} / \widetilde{E}$ is an embedding. This easily implies $\operatorname{deg}\left(\phi_{j}\right)=1$. Then, applying standard properties of linear systems on a $K 3$ surface, it follows that $\phi_{j}$ is a morphism and $X_{j}$ is normal with at most rational double points. Since $\left|L_{1}+L_{2}\right|=\left|\pi^{*} L\right|$ the map $\phi_{\widetilde{L}}$ has the same properties; since $\pi$ is étale and $\pi^{*} L \sim \widetilde{L} \sim \pi^{*} L^{\prime}$ the same is still true for $|L|,\left|L^{\prime}\right|$ on $X$. Consider

$$
\widetilde{X} \stackrel{\Psi}{\rightarrow} \mathbf{P}^{t} \times \mathbf{P}^{t} \stackrel{\sigma}{\rightarrow} \mathbf{P}^{\otimes}
$$

$\left(\sigma=\right.$ Segre embedding as in $\left.(3.2) ; \Psi=\left(\phi_{1} \times \phi_{2}\right)\right) . \sigma \cdot \Psi$ is the map defined by the vector space $\operatorname{Im}(\mu) \subset H^{0}\left(\mathscr{O}_{\widetilde{X}}(\widetilde{L})\right)$ where

$$
\mu: H^{0}\left(\mathscr{O}_{\widetilde{X}}\left(L_{1}\right)\right) \otimes H^{0}\left(\mathscr{O}_{\widetilde{X}}\left(L_{2}\right)\right) \rightarrow H^{0}\left(\mathscr{O}_{\widetilde{X}}(\widetilde{L})\right)
$$

is the multiplication map. First we want to show that $\mu$ is surjective. Observe that $|\widetilde{L}-\widetilde{E}|$ has no fixed components (it contains $R_{1}+\left|L_{2}\right|$ and $\left|L_{1}\right|+R_{2}$ ) and positive self-intersection. Therefore $h^{1}\left(\mathscr{O}_{\widetilde{X}}(\widetilde{L}-\widetilde{E})\right)=0$ and the sequence

$$
0 \rightarrow H^{0}\left(\mathscr{O}_{\widetilde{X}}(\widetilde{L}-\widetilde{E})\right) \rightarrow H^{0}\left(\mathscr{O}_{\widetilde{X}}(\widetilde{L})\right) \rightarrow H^{0}\left(\mathscr{O}_{\widetilde{E}}(\widetilde{L})\right) \rightarrow 0
$$


is exact. Let $T=H^{0}\left(\mathscr{O}_{\widetilde{X}}\left(L_{1}\right)\right) \otimes H^{0}\left(\mathscr{O}_{\widetilde{X}}\left(L_{2}\right)\right), T_{\widetilde{E}}=H^{0}\left(\mathscr{O}_{\widetilde{E}}\left(L_{1}\right)\right) \otimes H^{0}\left(\mathscr{O}_{\widetilde{E}}\left(L_{2}\right)\right)$; using (4.6) we get a homomorphism of exact sequences

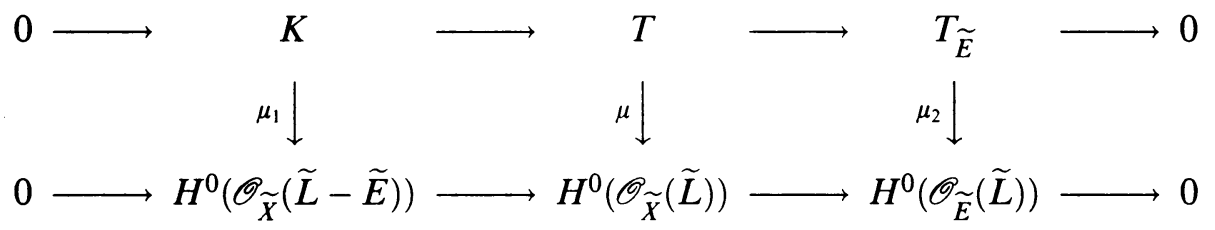

where $\mu_{1}, \mu_{2}$ are the induced multiplication maps. The surjectivity of $\mu_{2}$ for two very ample line bundles $\mathscr{O}_{\widetilde{E}}\left(L_{j}\right)$ on an elliptic curve is standard. To finish we must show that $\mu_{1}$ is surjective. Observe that $\operatorname{dim} H^{0}\left(\mathscr{O}_{\widetilde{X}}(\widetilde{L}-\widetilde{E})\right)=2 t$, $\operatorname{dim} K=2 t+1$. Fix $h_{j} \in H^{0}\left(\mathscr{O}_{\widetilde{X}}\left(L_{j}\right)\right), h_{j} \neq 0$ and vanishing on $\widetilde{E}$. Then $K$ is spanned by the vector spaces

$$
K_{1}=\left\langle h_{1}\right\rangle \otimes H^{0}\left(\mathscr{O}_{\widetilde{X}}\left(L_{2}\right)\right), \quad K_{2}=H^{0}\left(\mathscr{O}_{\widetilde{X}}\left(L_{1}\right)\right) \otimes\left\langle h_{2}\right\rangle .
$$

Therefore: $w \in \operatorname{Ker}\left(\mu_{1}\right) \Rightarrow\left(w=h_{1} \otimes s_{2}+s_{1} \otimes h_{2}\right.$ and $\left.\operatorname{div}\left(h_{1} s_{2}\right)=\operatorname{div}\left(h_{2} s_{1}\right)\right) \Rightarrow$ $\widetilde{E}+\operatorname{div}\left(s_{2}\right)=\widetilde{E}+\operatorname{div}\left(s_{1}\right)$. Since $R_{1}, R_{2}$ are disjoint and we can choose $\widetilde{E}$ irreducible, it follows $\operatorname{div}\left(s_{j}\right)=E_{j}+R_{j} ;\left(E_{j} \in|\widetilde{E}|\right)$. Hence $\operatorname{Ker}\left(\mu_{1}\right)$ is contained in $H^{0}\left(\mathscr{O}_{\widetilde{X}}\left(L_{1}-R_{1}\right)\right) \otimes H^{0}\left(\mathscr{O}_{\widetilde{X}}\left(L_{2}-R_{2}\right)\right) \cong H^{0}\left(\mathscr{O}_{\widetilde{X}}(\widetilde{E})\right) \otimes H^{0}\left(\mathscr{O}_{\widetilde{X}}(\widetilde{E})\right)$. The multiplication map on the latter vector space has 1-dimensional kernel. Hence $\operatorname{dim} \operatorname{Ker}\left(\mu_{1}\right)=1$ and $\mu, \mu_{1}$ are surjective. Now it is quite easy to reconstruct diagram (4.4) for the pair $(X, L)$ : since $\mu$ is surjective $\operatorname{Ker} \mu$ has codimension $h^{0}\left(\mathscr{O}_{\widetilde{X}}(\widetilde{L})\right)=4 t$ in $V^{*} \otimes V^{*}=H^{0}\left(\mathscr{O}_{\widetilde{X}}\left(L_{1}\right)\right) \otimes H^{0}\left(\mathscr{O}_{\widetilde{X}}\left(L_{2}\right)\right)$.

Moreover $\tilde{X}_{\widetilde{L}}$ spans $\mathbf{P}(K)$, with $K=\operatorname{Ker}(\mu)^{\perp}=\{u \in V \otimes V / h(u)=0$, $h \in \operatorname{Ker}(\mu)\}$. Since $i^{*} L_{1} \sim L_{2}$ we can assume that $i$ is induced by $I_{t}$ as in (3.3) and that $\mathbf{P}(K)$ is an invariant space of $I_{t}$. Let $j: K^{*} \rightarrow K^{*}$ be the involution induced by $I_{t}$ on $K^{*}$ then, under the restriction isomorphism $K^{*} \cong H^{0}\left(\mathscr{O}_{\widetilde{X}}(\widetilde{L})\right)$, the eigenspaces of $j$ are $\pi^{*} H^{0}\left(\mathscr{O}_{X}(L)\right), \pi^{*} H^{0}\left(\mathscr{O}_{X}\left(L^{\prime}\right)\right)$. Therefore, with the same notations of $(3.4)$, we have $p_{+}(\tilde{X})=X_{L}$ or $X_{L^{\prime}}$, $\left(p_{-}(\tilde{X})=X_{L}\right.$ or $\left.X_{L^{\prime}}\right)$. This implies that the condition (1) of (4.3) is satisfied up to showing $\pi^{*} H^{0}\left(\mathscr{O}_{X}(L)\right)=+1$ eigenspace of $i$ : we omit for brevity the proof of this last fact. The surjectivity of $\mu$ implies that condition (2) of (4.3) is also satisfied.

(4.8) Corollary. $h^{1}\left(\mathscr{O}_{X}\left(L^{\prime}-2 E\right)\right)=0$.

Proof. Observe that $h^{1}\left(\mathscr{O}_{X}\left(L^{\prime}-2 E\right)\right)=h^{1}\left(\mathscr{O}_{X}(-R)\right)$ by Serre duality and that the condition $h^{1}\left(\mathscr{O}_{X}(-R)\right)=0$ is equivalent to $h^{0}\left(\mathscr{O}_{R}\right)=1$.

Let $C H^{*}\left(G_{t}\right)$ be the Chow ring of $G_{t}$; we recall that $C H^{2}\left(G_{t}\right)$ is isomorphic to $\mathbf{Z}^{2}$ and generated by

$$
\begin{aligned}
& \sigma_{11}=\text { class of }\left\{l \in G_{t} / l \subset H\right\}, \quad(H \text { a given hyperplane }), \\
& \sigma_{20}=\left\{l \in G_{t} / l \cap M \neq \varnothing\right\}, \quad(M \text { a given codimension } 3 \text { subspace }) .
\end{aligned}
$$

Therefore, by the intersection pairing, $C H^{2}\left(G_{t}\right) \cong C H^{2 t-4}\left(G_{t}\right)$ and the rational equivalence class of a surface $S$ in $G_{t}$ is $\left(\sigma_{11} \cdot S, \sigma_{20} \cdot S\right)$.

(4.9) Corollary. The rational equivalence class of $X_{L^{\prime}}$ is $(t, 3 t-2)$. 
Proof. Let $h_{1}, h_{2}$ be the obvious generators of $C H^{*}\left(\mathbf{P}^{t} \times \mathbf{P}^{t}\right)$; an easy exercise shows that, under the homomorphism of Chow rings

$$
p_{-}^{*}: C H^{*}\left(G_{t}\right) \rightarrow C H^{*}\left(\mathbf{P}^{t} \times \mathbf{P}^{t}\right),
$$

$p_{-}^{*} \sigma_{20}=h_{1}^{2}+h_{2}^{2}+h_{1} h_{2}$ and $p_{-}^{*} \sigma_{11}=h_{1} h_{2}$. On the other hand one computes $\widetilde{X}_{\widetilde{L}} \sim(2 t-2)\left(h_{1}^{t-2} h_{2}^{t}+h_{1}^{t} h_{2}^{t-2}\right)+2 t\left(h_{1} h_{2}\right)^{t-1}$. Hence $\sigma_{11} \cdot X_{L^{\prime}}=\frac{1}{2} p_{-}^{*} \sigma_{11} \cdot \widetilde{X}_{\widetilde{L}}=t$, $\sigma_{20} \cdot X_{L^{\prime}}=\frac{1}{2} p_{-}^{*} \sigma_{20} \cdot \widetilde{X}_{\widetilde{L}}=3 t-2$.

Let $G_{t}^{*}$ be the dual grassmannian of $G_{t}$ and $g: G_{t}^{*} \rightarrow \mathbf{P}=\mathbf{P} H^{0}\left(\mathscr{O}_{G_{t}}(1)\right)$ the map sending $M \in G_{t}^{*}$ in the codimension 1 Schubert cycle $\sigma_{M}=\{l \in$ $\left.G_{t} / l \cap M \neq \varnothing\right\} . g$ is just the Plücker embedding of $G_{t}^{*}$.

Observe that $\sigma_{M}$ is ruled by a pencil of codimension 2 Schubert cycles of class $\sigma_{11}$ : the elements of this pencil are the grassmannians of lines of the hyperplanes through $M$.

Assume $\sigma_{M}$ does not contain $X_{L^{\prime}}$ : since $\sigma_{11} \cdot X_{L^{\prime}}=t$ the hyperplane section $L^{\prime}=\sigma_{M} \cdot X_{L^{\prime}}$ is a $t$-gonal curve. Let $r: H^{0}\left(\mathscr{O}_{G_{t}}(1)\right) \rightarrow H^{0}\left(\mathscr{O}_{X}\left(L^{\prime}\right)\right)$ be the restriction map; of course $r$ induces a linear projection $p: \mathbf{P} \rightarrow \mathbf{P} H^{0}\left(\mathscr{O}_{X}\left(L^{\prime}\right)\right)$ of center $\left|\mathscr{I}_{X}(1)\right|,\left(\mathscr{J}_{X}=\right.$ ideal of $X_{L^{\prime}}$ in $\left.G_{t}\right)$. We expect that, for general $X_{L^{\prime}}, p / G_{t}$ is a birational morphism. However: assume $p / G_{t}$ is generically finite, then

(4.10) Corollary. $\left|\mathscr{O}_{X}\left(L^{\prime}\right)\right|$ contains a codimension 1 family of $t$-gonal curves.

For any polarization $L$ on $X$ let us define

(4.11) $\mathscr{E}_{L}(s)=\left\{E / E=\right.$ isolated elliptic curve, $E \cdot L=s, h^{1}\left(\mathscr{O}_{X}\left(L^{\prime}-2 E\right)\right)=$ $0\} \quad\left(L^{\prime}=L+K_{X}\right)$, then

(4.12) Theorem. Let $L$ be a polarization on $X$ as in (4.2), $L^{2}=4 t-2 \geq 10$ : Assume $\mathscr{E}_{L}(t) \neq \varnothing$, then the following conditions are equivalent:

(1) The pair $(X, L)$ fits in a Reye diagram of index $t$,

(2) $h^{1}\left(\mathscr{O}_{X}\left(E+E^{\prime}-L\right)\right)=1$ for all $E \in \mathscr{E}_{L}(t)$,

(3) $h^{1}\left(\mathscr{O}_{X}\left(E+E^{\prime}-L\right)\right)=1$ for some $E \in \mathscr{E}_{L}(t)$,

(4) $L \sim 2 E+R$ with $E=$ isolated elliptic curve, $R=$ indecomposable nodal cycle, $E \cdot R=t$.

Proof. (1) $\Rightarrow(2)$ : let $E \in \mathscr{E}_{L}(t)$, first we show $h^{0}\left(\mathscr{O}_{X}\left(L^{\prime}-E-E^{\prime}\right)\right)>0$. By assumption $X_{L^{\prime}}$ fits in diagram (4.4), therefore, with the same notations used there, we have that $\widetilde{E}=\left(p_{-} / \widetilde{X}\right)^{*}(E)$ is a degree $2 t$ elliptic curve which is invariant with respect to the involution $I_{t}(x, y)=(y, x)$. Let $\widetilde{E}_{i}$ be the projection of $\widetilde{E}$ on the $i$ th factor of $\mathbf{P}^{t} \times \mathbf{P}^{t}$ then $\operatorname{deg}\left(\widetilde{E}_{i}\right)=t$ and $\widetilde{E}_{i}$ is contained in a hyperplane. This means $\widetilde{E} \subset\left\{x_{0}=y_{0}=0\right\}$ where $\left(x_{0}: \cdots: x_{t}\right) \times\left(y_{0}: \cdots: y_{t}\right)$ are suitable coordinates on $\mathbf{P}^{t} \times \mathbf{P}^{t}$. The same holds for $\widetilde{E}^{\prime}=\left(p_{-} / \widetilde{X}\right)^{*}\left(E^{\prime}\right)$. Assume we have again $\widetilde{E}^{\prime} \subset\left\{x_{0}=y_{0}=0\right\}$. Let $H=\left\{x_{0} y_{0}=0\right\}$; note that, by (4.3)(2), $\widetilde{X}$ is not in $H$ and that $H$ is +1-invariant with respect to $I_{t}$. Therefore $H \cdot \tilde{X}$ would be the pull back of an element of $\left|\mathscr{O}_{X}(L)\right|$ and $h^{0}\left(\mathscr{O}_{X}\left(L-E-E^{\prime}\right)\right)>0$. Since $h^{0}\left(\mathscr{O}_{X}\left(L-E-E^{\prime}\right)\right)=h^{1}\left(\mathscr{O}_{X}\left(L^{\prime}-2 E\right)\right)=0$ this is impossible. Hence, up to changing coordinates, we can assume $\widetilde{E} \subset$ $\left\{x_{0}=y_{0}=0\right\}, \widetilde{E}^{\prime} \subset\left\{x_{1}=y_{1}=0\right\}$. Finally consider $\tilde{\sigma}=\left\{x_{0} y_{1}-x_{1} y_{0}=0\right\}$ and assume $\tilde{X} \subset \tilde{\sigma}$. Then the rational function on $\tilde{X}: \tilde{f}=x_{1} / x_{0}=y_{1} / y_{0}$ 
is +1 -invariant with respect to the fixed-point-free involution of $\tilde{X}$. On the other hand $\tilde{f}=\left(p_{-} / \tilde{X}\right)^{*} f$ where $\operatorname{div}(f)=E-E^{\prime}$. As is well known the pull back of $f$ is -1 -invariant, hence $\widetilde{X}$ is not in $\tilde{\sigma}$. Note that $\tilde{\sigma}=p_{-}^{*}(\sigma)$, where $\sigma$ is a hyperplane section of $G_{t}$ (more precisely: a codimension 1 Schubert cycle). Hence $X \cdot \sigma=R+E+E^{\prime}, h^{0}\left(\mathscr{O}_{X}\left(L^{\prime}-E-E^{\prime}\right)\right)>0$. To show $h^{1}\left(\mathscr{O}_{X}\left(E+E^{\prime}-L\right)\right)=1$ first observe that $h^{0}\left(\mathscr{O}_{X}(R)\right)=h^{1}\left(\mathscr{O}_{X}\left(E+E^{\prime}-L\right)\right)$. Moreover $h^{1}\left(\mathscr{O}_{X}(-R)\right)=h^{1}\left(\mathscr{O}_{X}\left(L^{\prime}-2 E\right)\right)=0 \Rightarrow h^{0}\left(\mathscr{O}_{R}\right)=1 \Rightarrow R$ is connected. Then, since $p_{a}(R)=0, h^{0}\left(\mathscr{O}_{X}(R)\right)=1$.

(2) $\Rightarrow(3)$ : obvious.

(3) $\Rightarrow(4): h^{1}\left(\mathscr{O}_{X}\left(E+E^{\prime}-L\right)\right)=1 \Rightarrow L^{\prime}-E-E^{\prime} \sim R$, with $R$ effective, $p_{a}(R)=0, E \cdot R=t$. The condition $h^{0}\left(\mathscr{O}_{R}\right)=1$ can be shown as above. $(4) \Rightarrow(1)$ is Proposition 4.5 .

(4.13) Definition. Let $L$ be a polarization on $X$ as in (4.2). Assume (i) $\mathscr{E}_{L}(t)$ $\neq \varnothing$, (ii) the equivalent conditions of Theorem (4.13) are satisfied. Then we say that $L^{\prime}=L+K_{X}$ is a Reye polarization of index $t$ and $X_{L^{\prime}}$ a Reye congruence of index $t$.

\section{REYE CONSTRUCTIONS}

The original construction of Reye congruences in $G_{3}$ was given as follows:

(5.1) Let $W$ be a general 3-dimensional linear system of quadrics of $\mathbf{P}^{3}$ and

$$
X=\left\{l \in G_{3} / l \text { is included in two quadrics of } W\right\}
$$

then $X$ is a Reye congruence and, conversely, all Reye congruences arise in this way. At the end we want to point out that (5.1) generalizes again to Reye congruences in $G_{t}$ :

(5.2) Theorem. Let $X$ be a Reye congruence of index $t$. Then there exists a linear system $W$ of quadrics of $\mathbf{P}^{t}$ such that

(i) $\operatorname{dim} W=\left(\begin{array}{l}t \\ 2\end{array}\right)$;

(ii) $X=\left\{l \in G_{t} \cap \mathbf{P}^{2 t-1} / \operatorname{codim}\left(W_{l}, W\right)=2\right\}$;

where $W_{l}=\{Q \in W / l \subset Q\}, \mathbf{P}^{2 t-1}=$ linear span of $X$ in the Plücker space of $G_{t}$.

To show (5.2) we first consider the following situation: $E=$ elliptic curve; $\mathscr{O}_{E}\left(L_{1}\right), \mathscr{O}_{E}\left(L_{2}\right)=$ line bundles of degree $t \geq 3$ on $E$. Assume $L_{1} \nsim L_{2}$ and consider

$$
E \stackrel{\gamma_{1} \times \gamma_{2}}{\rightarrow} \mathbf{P}^{t-1} \times \mathbf{P}^{t-1} \stackrel{\sigma}{\rightarrow} \mathbf{P}^{t^{2}-1}
$$

with $\sigma=$ Segre inclusion and $\gamma_{j}=\operatorname{map}$ associated to $\left|L_{j}\right|$. Let $E^{\prime}=$ $\sigma \cdot\left(\gamma_{1} \times \gamma_{2}\right)(E)$, then

(5.3) Lemma. Let $\Lambda$ be the $(2 t-1)$-space spanned by $E^{\prime}$ in the ambient space of $\mathbf{P}^{t-1} \times \mathbf{P}^{t-1}$ then $E^{\prime}=\Lambda \cap \mathbf{P}^{t-1} \times \mathbf{P}^{t-1}$.

Proof. For brevity we will leave some details as an exercise. Consider the multiplication map $\mu: H^{0}\left(\mathscr{O}_{E}\left(L_{1}\right)\right) \otimes H^{0}\left(\mathscr{O}_{E}\left(L_{2}\right)\right) \rightarrow H^{0}\left(\mathscr{O}_{E}\left(L_{1}+L_{2}\right)\right)$. Since $\mu$ is surjective $\operatorname{dim} \operatorname{Ker}(\mu)=t^{2}-2 t$ and $E^{\prime}$ is contained in $t^{2}-2 t$ linearly independent hyperplanes of $\mathbf{P}^{t^{2}-1}$. Let $\Lambda$ be the intersection of them and

$$
Z=\Lambda \cap \mathbf{P}^{t-1} \times \mathbf{P}^{t-1} \text {. }
$$

We have to show that $E^{\prime}=Z$ : this is done by induction on $t$. 
$t=3$ : in this case $\Lambda=\mathbf{P}^{5}, E^{\prime}=$ sextic curve. Note that the Segre variety $\mathbf{P}^{2} \times \mathbf{P}^{2}$ is a 4 -fold of degree 6 in $\mathbf{P}^{8}$. Assume $E^{\prime} \subset Z$, then, by Bézout theorem and its corollaries, $\operatorname{dim} Z \geq 2$ and there exists an irreducible component $Y$ of $Z$ which contains properly $E^{\prime}$. Let $h_{j}(j=1,2)$ be the obvious generators of $C H^{*}\left(\mathbf{P}^{2} \times \mathbf{P}^{2}\right), \pi_{j}: \mathbf{P}^{2} \times \mathbf{P}^{2} \rightarrow \mathbf{P}^{2}$ the canonical projections. If $\operatorname{dim} Y=2$ then $\operatorname{deg}(Y) \leq 5 ;$ assume $\operatorname{dim}(Y)=2, \operatorname{deg}(Y)=5$ or $\operatorname{dim}(Y)=3$ then $Y \sim h_{j}^{2}+2 h_{1} h_{2}$ or $Y \sim h_{j}$. In both cases one can check that $\pi_{j}(Y)$ is a line. Since $\pi_{j}\left(E^{\prime}\right) \subset \pi_{j}(Y)$ it follows that $\pi_{j}\left(E^{\prime}\right)$ does not span $\mathbf{P}^{2}$ : a contradiction. Hence $\operatorname{dim}(Y)=2, \operatorname{deg}(Y)=4$; this implies $Y \sim h_{1}^{2}+h_{2}^{2}+h_{1} h_{2}$, $Y=$ Veronese surface. Hence $Y$ is the graph of a projective isomorphism $f: \mathbf{P}^{2} \rightarrow \mathbf{P}^{2}$ such that $f\left(\pi_{1}\left(E^{\prime}\right)\right)=\pi_{2}\left(E^{\prime}\right)$. Since $L_{1} \nsim L_{2}$ this is again a contradiction. Therefore $E^{\prime}=\Lambda \cap \mathbf{P}^{2} \times \mathbf{P}^{2}$.

$t>3$ : let $p=\left(p_{1}, p_{2}\right) \in E^{\prime}, \phi_{i}: \mathbf{P}^{t-1} \rightarrow \mathbf{P}^{t-2}$ the projection from $p_{i}$ and

$$
\phi=\phi_{1} \times \phi_{2}: \mathbf{P}^{t-1} \times \mathbf{P}^{t-1} \rightarrow \mathbf{P}^{t-2} \times \mathbf{P}^{t-2} .
$$

The fundamental locus of $\phi$ is $\left\{p_{1}\right\} \times \mathbf{P}^{t-1} \cup \mathbf{P}^{t-1} \times\left\{p_{2}\right\}$. Let $\Gamma$ be the linear span of this set,

$$
\gamma: \mathbf{P}^{t^{2}-1} \rightarrow \mathbf{P}^{(t-1)^{2}-1}
$$

the projection from $\Gamma$; then $\sigma \cdot \phi=\gamma / \mathbf{P}^{t-1} \times \mathbf{P}^{t-1}$ (with $\sigma=$ Segre embedding of $\left.\mathbf{P}^{t-2} \times \mathbf{P}^{t-2}\right)$. Now observe the following:

(i) $\phi / E^{\prime}=\psi_{1} \times \psi_{2}$ with $\psi_{i}=$ morphism associated to $\left|L_{i}-p\right|$;

(ii) $\gamma / \Lambda$ is the projection from $l=$ tangent line to $E^{\prime}$ at $p$ and $\Gamma \cap \Lambda=l$.

Let $\Lambda^{\prime}=\gamma(\Lambda), E^{\prime \prime}=\phi\left(E^{\prime}\right)$; then $\Lambda^{\prime}$ is the linear span of $E^{\prime \prime}$ and, by (i) and the induction

$$
E^{\prime \prime}=\Lambda^{\prime} \cap \mathbf{P}^{t-2} \times \mathbf{P}^{t-2} .
$$

Therefore, to complete the proof of our statement, it remains only to show that

$$
\forall q \in E^{\prime} \quad \overline{(\gamma / \Lambda)^{*}(\phi(q))} \cdot \overline{\phi^{*}(\phi(q))}=p+q
$$

(the overline denoting Zariski closure). Now $\overline{(\gamma / \Lambda)^{*}(\phi(q))}=\alpha_{q}=$ plane containing $l$ and $q$; while $\overline{\phi^{*}(\phi(q))}=l_{1} \times l_{2}$, with $l_{i}=$ line through $p_{i}$. The Segre map embeds $l_{1} \times l_{2}$ in $\mathbf{P}^{t^{2}-1}$ as a smooth quadric surface $S_{q}$. Let $P_{q}$ be the 3-space of $S_{q}, l_{q}$ the line joining $p$ to $q$; then $l_{q} \subseteq \alpha_{q} \cap S_{q}$. Clearly (5.4) holds if and only if: (1) $l_{q}$ is not in $S_{q}$, (2) $\alpha_{q}$ is not in $P_{q}$ (i.e. $l_{q}=\alpha_{q} \cap P_{q}$ ).

Assume $l_{q} \subset S_{q}$ then $l_{q}$ is $\left\{p_{1}\right\} \times l_{1}$ or $l_{1} \times\left\{p_{2}\right\}$ and $\pi_{1}\left(l_{q}\right)$ or $\pi_{2}\left(l_{q}\right)$ is a point; since $l_{q}$ is bisecant to $E^{\prime}$ and $\pi_{i}: E^{\prime} \rightarrow \mathbf{P}^{t-1}$ is an embedding we get a contradiction. Hence (1) holds.

Assume $l \subset P_{q}$; by (ii) $l \subset \Gamma \cap P_{q}$ which is just the plane of the lines $\left\{p_{1}\right\} \times l_{2}, l_{1} \times\left\{p_{2}\right\}$ (i.e. the tangent plane to $S_{q}$ at $p$ ). Hence the image of $l$ by the tangent map $d(\pi)_{p}$ is $l_{i}$. This implies $l_{i}=$ tangent line to $\pi_{i}\left(E^{\prime}\right)$ at $p_{i}$ and $p=q$. Therefore $p \neq q \Rightarrow l$ not in $P_{q} \Rightarrow \alpha_{q}$ not in $P_{q}$ and (2) holds for $p \neq q$. After some tedious remarks one can show (2) also in the case $p=q$.

(5.5) Remark. With some more effort one can show the same for any divisor of canonical type. 
Now let $X$ be an Enriques surface, $L^{\prime}=L+K_{X}$ a Reye polarization of index $t$ on it; by $(4.4)$ the pair $(X, L)$ defines the Reye diagram

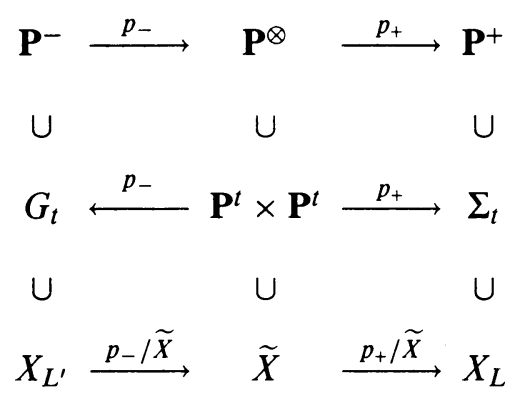

we have

(5.6) Lemma. Let $\tilde{\Lambda}$ be the linear span of $\tilde{X}$ in $\mathbf{P}^{\otimes}$, then $\tilde{X}=\widetilde{\Lambda} \cap \mathbf{P}^{t} \times \mathbf{P}^{t}$.

Proof. Let $\pi_{j}: \mathbf{P}^{t} \times \mathbf{P}^{t} \rightarrow \mathbf{P}^{t}$ be the canonical projections $(j=1,2), X_{j}=$ $\pi_{j}(\tilde{X})$. By (4.12) $L \sim 2 E+R$, with $E=$ isolated elliptic curve, $R=$ indecomposable nodal cycle and $E \cdot R=t$. As in the proof of (4.5) consider $|\widetilde{E}|=\left|\pi^{*} E\right|, R_{1}+R_{2}=\pi^{*} R$ : we know that, for the fixed points free involution $i$ on $\tilde{X}, i=I_{t} / \tilde{X}$, where $I_{t}$ is the involution on $\mathbf{P}^{t} \times \mathbf{P}^{t}$ sending $(x, y)$ in $(y, x)$; moreover $\mathscr{O}_{X_{j}}(1) \cong \mathscr{O}_{\widetilde{X}}\left(\widetilde{E}+R_{j}\right)$. Therefore we can fix projective coordinates $\left(x_{0} \cdots x_{t}\right) \times\left(y_{0} \cdots y_{t}\right)$ on $\mathbf{P}^{t} \times \mathbf{P}^{t}$ such that $i=I_{t} / \tilde{X}$ and the divisor associated to the rational function $x_{0} / x_{1}$ on $X_{1}$ is $F_{0}-F_{1},\left(F_{0}, F_{1} \in|\widetilde{E}|\right)$. Since $x_{0} / x_{1}$ is anti-invariant we have $-x_{0} / x_{1}=i^{*}\left(x_{0} / x_{1}\right)=y_{0} / y_{1}$, so that $\widetilde{X} \subset H$ where the equation of $H$ in $\mathbf{P}^{t} \times \mathbf{P}^{t}$ is $x_{0} y_{1}+x_{1} y_{0}=0 . H$ contains the pencil of divisors

$$
H_{z}=\left\{z_{0} x_{0}+z_{1} x_{1}=z_{0} y_{0}-z_{1} y_{1}=0\right\}
$$

with $z=\left(z_{0}, z_{1}\right) \in \mathbf{P}^{1}$. Observe that $H_{z} \cap \widetilde{X} \supset \widetilde{E}_{z}$ for some $\widetilde{E}_{z} \in|\widetilde{E}|$ and that $H_{z}=\mathbf{P}^{t-1} \times \mathbf{P}^{t-1}$. Then consider $\mathscr{O}_{\widetilde{E}_{z}}\left(L_{j}\right)=\mathscr{O}_{\widetilde{E}_{z}}\left(\widetilde{E}+R_{j}\right):$ the embedding of $\widetilde{E}_{z}$ in $H_{z}$ is exactly the one described above in Lemma (5.3); moreover, applying standard exact sequences, one easily shows $\mathscr{O}_{\widetilde{E}_{z}}\left(L_{1}\right) \neq \mathscr{O}_{\widetilde{E}_{z}}\left(L_{2}\right)$. Therefore, by Lemma (5.4), we obtain

$$
\widetilde{E}_{z}=\Lambda_{z} \cap H_{z}
$$

$\Lambda_{z}$ being the linear span of $\widetilde{E}_{z}$. Finally $\widetilde{E}_{z}=\tilde{\Lambda} \cap H_{z} \quad(\forall z) \Rightarrow \tilde{X}=\tilde{\Lambda} \cap H \Rightarrow$ $\widetilde{X}=\tilde{\Lambda} \cap \mathbf{P}^{t} \times \mathbf{P}^{t}$ (because $H$ is a hyperplane section of $\mathbf{P}^{t} \times \mathbf{P}^{t}$ ).

Let $\mathscr{I}_{\widetilde{X}}$ be the ideal of $\widetilde{X}$ in $\mathbf{P}^{t} \times \mathbf{P}^{t}, J$ the ideal of $\widetilde{E}_{z}$ in $H_{z}$; consider the natural restriction map

$$
r: H^{0}\left(\mathscr{I}_{\widetilde{X}}(1)\right) \rightarrow H^{0}(\mathscr{T}(1))
$$

By Lemma (5.3), to show $\widetilde{E}_{z}=\tilde{\Lambda} \cap H_{z}$, it suffices to show that $r$ is surjective. For this let $\mathscr{I}_{z}$ be the ideal of $H_{z}$ in $\mathbf{P}^{t} \times \mathbf{P}^{t}$; observe that $H^{1}\left(\mathscr{I}_{\widetilde{X}}(1)\right)=$ $H^{1}\left(\mathscr{J}_{z}(1)\right)=0$ because $\tilde{X}$ and $H_{z}$ are linearly normal. For the same reason 
$H^{1}\left(\mathscr{O}_{\widetilde{X}}(\widetilde{L}-\widetilde{E})\right)=0$. Then we have a commutative diagram

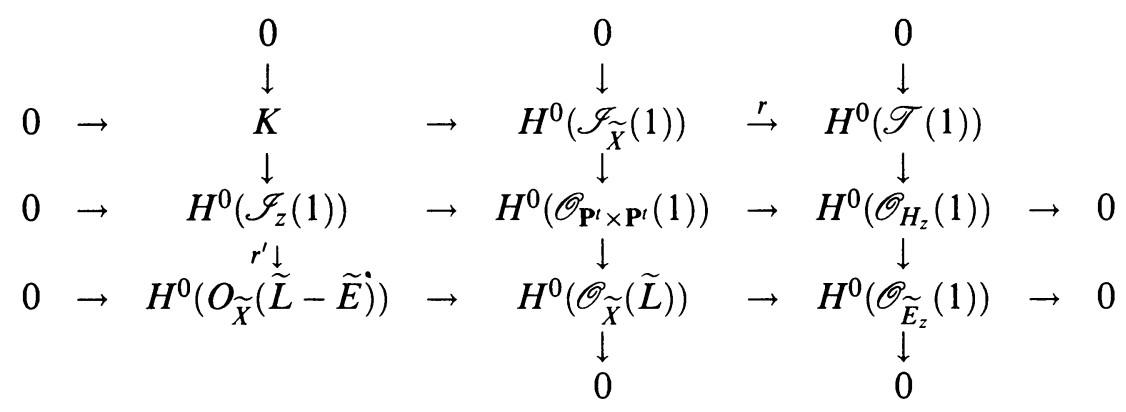

the second and third rows (columns) of which are exact. By the snake lemma $r$ is surjective iff $r^{\prime}$ is surjective. One easily computes $h^{0}\left(\mathscr{F}_{z}(1)\right)=2 t+1$, $h^{0}\left(\mathscr{O}_{\widetilde{X}}(\widetilde{L}-\widetilde{E})\right)=2 t$. Let $s=x_{0} y_{1}+x_{1} y_{0}, \operatorname{since} \operatorname{div}(s)=H, s$ belongs to $K$.

Let $s^{\prime}$ be another element of $K$ : as for any element of $H^{0}\left(\mathscr{I}_{z}(1)\right)$ we can write $s^{\prime}=\left(z_{0} x_{0}+z_{1} x_{1}\right) A+\left(z_{0} y_{0}-z_{1} y_{1}\right) B$ (with $A(B)=$ linear form in $\left(y_{0} \cdots y_{t}\right.$ ) (in $\left(x_{0} \cdots x_{t}\right)$ ). Let $H^{\prime}=\operatorname{div}\left(s^{\prime}\right)$; then $H_{z}$ moves in a pencil $\left\{H_{t}^{\prime}, t \in \mathbf{P}^{1}\right\}$ on $H^{\prime}$ having the same properties of the pencil $\left\{H_{z}\right\}$ of $H$. In particular, changing $t$ by $z$, we have $\widetilde{E}_{z} \subset H_{z}^{\prime} \forall z$. Let $h_{1, z}, h_{2, z}$ be the equations of $H_{z}^{\prime}$, then, for the two projections of $\widetilde{E}_{z}$, we have $\pi_{1}\left(\widetilde{E}_{z}\right) \subset$ $\left\{h_{1, z}=0\right\} \cap\left\{z_{0} x_{0}+z_{1} x_{1}=0\right\}$ and $\pi_{2}\left(\widetilde{E}_{x}\right) \subset\left\{h_{2, z}=0\right\} \cap\left\{z_{0} y_{0}-z_{1} y_{1}=0\right\}$. Now there is a unique hyperplane containing $\pi_{j}\left(\widetilde{E}_{z}\right)$ because

$$
h^{0}\left(\mathscr{O}_{X_{j}}(1) \otimes \mathscr{O}_{X_{j}}\left(-\widetilde{E}_{x}\right)\right)=h^{0}\left(\mathscr{O}_{X_{j}}(R)\right)=1 .
$$

Therefore $H_{z}=H_{z}^{\prime} \quad \forall z, H=H^{\prime}$ and $s^{\prime}=c s$ for some constant $c$. Hence $\operatorname{dim}(K)=1$ and the proof is complete.

Finally we can give a

Proof of (5.2). By (5.6) $\tilde{X}=\tilde{\Lambda} \cap \mathbf{P}^{t} \times \mathbf{P}^{t}$. Let $\Lambda_{+}=p_{+}(\tilde{\Lambda}), \Lambda_{-}=p_{-}(\tilde{\Lambda})$. By the Reye diagram $X_{L^{\prime}} \subset \Lambda_{-} \cap G_{t} \subset \mathbf{P}^{-}, X_{L} \subset \Lambda_{+} \cap \Sigma_{t} \subset \mathbf{P}^{+}$and $\operatorname{dim}\left(\Lambda_{-}\right)=$ $\operatorname{dim}\left(\Lambda_{+}\right)=2 t-1$. We denote by $W$ the linear system of hyperplanes of $\mathbf{P}^{+}$ containing $\Lambda_{+}$. Clearly $\operatorname{dim}(W)=\operatorname{dim}\left(\mathbf{P}^{+}\right)-\operatorname{dim}\left(\Lambda_{-}\right)-1=\left(\begin{array}{l}t \\ 2\end{array}\right)$.

Recall, (3.4), that $\mathbf{P}^{+}=\mathbf{P}\left(\operatorname{Sym}^{2} V^{*}\right)=\mathbf{P} H^{0}\left(\mathscr{O}_{\mathbf{P}(V)}(2)\right)$ so that $W$ is a linear system of quadrics of $\mathbf{P}^{t}=\mathbf{P}(V)$. Now identify (canonically) $\operatorname{Sym}^{2}\left(V^{*}\right)$ to the vector space of symmetric bilinear maps on $V \times V$; then denote by $\widehat{W}$ the subvector space whose projectivization is $W$. Let $x \in \mathbf{P}^{t} \times \mathbf{P}^{t}, x$ not in the diagonal. $x$ corresponds (up to scalars) to a pair $\left(u_{1}, u_{2}\right)$ of linearly independent vectors of $V$; moreover the line $u=p_{-}(x)$ is the projectivization of the vector space $U=\left\langle u_{1}, u_{2}\right\rangle$.

Observe that, by Lemma (5.6), $x \in \widetilde{X}$ if and only if $u \in \Lambda_{-}$and $q\left(u_{1}, u_{2}\right)=$ 0 for all symmetric bilinear maps $q \in \widehat{W}$. Let $\widehat{W}_{U}=\{q \in \widehat{W} / U$ is isotropic for $q\}$ : it is a standard exercise in linear algebra showing that $\operatorname{codim}\left(\widehat{W}_{U}, \widehat{W}\right) \leq 2$ if and only if there exist vectors $u_{1}, u_{2} \in U / q\left(u_{1}, u_{2}\right)=0 \quad \forall q \in \widehat{W}$. Hence $X_{L^{\prime}}=p_{-}(\widetilde{X})=\left\{u \in G_{t} \cap \Lambda_{-} / \operatorname{codim}\left(W_{u}, W\right) \leq 2\right\}$. Assume $\operatorname{codim}\left(W_{u}, W\right) \leq$ 1 (for some $u \in X_{L^{\prime}}$ ); then $W$ has a based point $p$ on $u$. Equivalently $(p, p)$ is contained in each hyperplane of $\mathbf{P}^{\otimes}$ which contains $\tilde{\Lambda}$ and is +1 -invariant 
with respect to $I_{t}$. On the other hand, since it is in the diagonal of $\mathbf{P}^{t} \times \mathbf{P}^{t}$, $(p, p)$ is in all -1 hyperplanes of $I_{t}$ Hence $(p, p) \in \tilde{\Lambda}$ and, by Lemma 5.6 , $(p, p) \in \tilde{X}$ : this is a contradiction because $I_{t} / \widetilde{X}$ is base-point-free. Hence $\operatorname{codim}\left(W_{u}, W\right)=2$ and the proof is complete.

\section{EXISTENCE OF REYE CONGRUENCES OF INDEX $t \geq 3$}

Now we want to check for which values of $t$ the Reye construction is possible. Let $X$ be a nodal Enriques surface,

$\mathscr{R}(X)=\{R \subset X / R=$ indecomposable nodal cycle $\}$

$\mathscr{E}(X)=\{E \subset X / E=$ isolated elliptic curve $\}$, and

$$
m: \mathscr{R}(X) \times \mathscr{E}(X) \rightarrow \mathbf{N}
$$

the intersection product. By (4.12) $X$ admits a Reye polarization of index $t$ if and only if $t \in \operatorname{Im}(m)$. In this section we show that actually $\operatorname{Im}(m)=\mathbf{N}$ on a general nodal $X$. To make precise what we mean by general we give the following

(6.2) Definition [10, vol. II]. A nodal Enriques surface $X$ is said to be generic if all the elements of $\mathscr{R}(X)$ have the same numerical equivalence class modulo $2 L, L=\operatorname{Num}(X)$.

(6.3) Definition. Let $X$ be a nodal Enriques surface, $C$ a Reye polarization on $X$, we will say that $C$ is good if $C \cdot R \geq 4 \forall R \in \mathscr{R}(X)$.

Note that $C$ is very ample because there is no $R \in \mathscr{R}(X)$ such that $R \cdot C$ $=0$. Moreover $[10$, vol. II]:

(6.4) $X$ is generic if and only if $X$ admits a good Reye polarization.

Finally it is known $[9,10]$ that a general (in sense of moduli) nodal $X$ is generic.

Let $C$ be a good Reye polarization on $X ; E_{i}, E_{i}^{\prime}$ the twenty plane cubics in the smooth Reye model $X_{C}$; it is well known (cf. proof of (3.11)) that

$$
C-E_{i}-E_{i}^{\prime} \sim R_{i}
$$

with $R_{i}=$ indecomposable nodal cycle, $C \cdot R_{i}=4$. Actually, since $C$ is good and $h^{0}\left(\mathscr{O}_{X}\left(C-R_{i}\right)\right)=1, R_{i}$ is a rational normal quartic curve in $\mathbf{P}^{4}$. Our program is to fix one of these curves, e.g.

$$
R=R_{1}
$$

and construct a sequence $\left\{F_{t}, t \geq 1\right\}$ of isolated elliptic curves such that $F_{t} \cdot R=$ $t$. First we construct such a sequence numerically: in the lattice $L=\operatorname{Num}(X)$ we consider all the sets $\left\{e_{1} \cdots e_{10}\right\}$ of isotropic vectors satisfying $e_{i} \cdot e_{j}=1-\delta_{i j}$, $\frac{1}{3} \sum e_{i} \in L$ as in (1.1). Let $c=\frac{1}{3} \sum e_{i}$, we will say that $c$ is a Fano vector. Let $l_{i j k}=c-e_{i}-e_{j}-e_{k}$, (with $i, j, k=$ distinct elements of $\{1 \cdots 10\}$ ); we define

$$
s_{i j k}: L \rightarrow L
$$

by $s_{i j k}(v)=v+\left(v \cdot l_{i j k}\right) l_{i j k}$. Since $l_{i j k}^{2}=-2 s_{i j k}$ is a reflection and $s_{i j k}\left(s_{i j k}(v)\right)$ $=v$. Let $c^{\prime}=s_{i j k}(c)$. Observe that $c^{\prime}$ has the following properties:

$$
c^{\prime}=2 c-e_{i}-e_{j}-e_{k} .
$$


Let $e_{m}^{\prime}=s_{i j k}\left(e_{m}\right)$, then $\left\{e_{1}^{\prime} \cdots e_{10}^{\prime}\right\}$ is again a set of isotropic vectors satisfying $(1.1)$, moveover

$$
\begin{gathered}
e_{m}^{\prime}=e_{m}, \quad m \neq i, j, k, \quad e_{i}^{\prime}=c-e_{j}-e_{k}, \\
e_{j}^{\prime}=c-e_{i}-e_{k}, \quad e_{k}^{\prime}=c-e_{i}-e_{j} .
\end{gathered}
$$

In particular $c^{\prime}=s_{i j k}(c)=\frac{1}{3} \sum e_{i}^{\prime}$ is still a Fano vector. Finally, $\forall v \in L$, consider the intersection numbers

$$
d=v \cdot c, \quad v_{i}=v \cdot e_{i}, \quad d^{\prime}=v \cdot c^{\prime}, \quad v_{i}^{\prime}=e_{i}^{\prime} \cdot v
$$

by definition of $s_{i j k}$ we have

$$
\begin{gathered}
d^{\prime}=2 d-v_{i}-v_{j}-v_{k}, \quad v_{i}^{\prime}=d-v_{j}-v_{k}, \quad v_{j}^{\prime}=d-v_{i}-v_{k}, \\
v_{k}^{\prime}=d-v_{i}-v_{j}, \quad v_{m}^{\prime}=v_{m} \quad(m \neq i, j, k) .
\end{gathered}
$$

Now we consider the following functions on $L$ :

$$
f=s_{178} \cdot s_{256} \cdot s_{234}, \quad g=s_{178} \cdot s_{256} \cdot s_{234} \cdot s_{1910}
$$

and

$$
h_{t}=g^{t-1} \cdot f, \quad t \geq 1 .
$$

Then we define in $L$ the elements

$$
c_{t}=h_{t}(c)=\frac{1}{3} \sum e_{i}^{t}, \quad f_{t}=e_{10}^{t}, \quad r=c-2 e_{1}
$$

$\left(e_{i}^{t}=h_{t}\left(e_{i}\right)\right)$. Let $v_{i}=r \cdot e_{i}$, one immediately computes $v_{1}=3, v_{i}=1$, $i \geq 2$. Then, applying repeatedly the previous formulae $(6.10)$, one computes with some pain:

$$
\begin{array}{ccc}
r \cdot e_{1}^{t}=2 t+2, \quad r \cdot e_{2}^{t}=2 t+1, & r \cdot e_{3}^{t}=\cdots=r \cdot e_{8}^{t}=t+1, \\
r \cdot e_{9}^{t}=r \cdot e_{10}^{t}=t \quad \text { and } & c_{t} \cdot r=4 t+3 .
\end{array}
$$

In particular it follows $r \cdot f_{t}=t$. Since $f_{t}^{2}=0, f_{t} \cdot e_{1}^{t}=1, f_{t}$ has the numerical properties of the class of an isolated elliptic curve on $X$. On the other hand $r^{2}=-2$ so that, numerically, $r$ can be the class of an indecomposable nodal cycle. Now we shall show that, for a given (nodal) Enriques surface $X$, one can construct in $\operatorname{Num}(X)$ elements $r, f_{t}$ which are represented by curves $R \in \mathscr{R}(X), F_{t} \in \mathscr{E}(X)$. Let

(6.15) Proposition. Let $X_{C}$ be a smooth Fano model; $E_{i}, E_{i}^{\prime}$ its twenty plane cubics. Fix three distinct $i, j, k \in\{1 \cdots 10\}$ and consider the linear system

$$
|H|=\left|2 C-E_{i}-E_{j}-E_{k}\right| \text {. }
$$

Assume there is no line in $X_{C}$, then $H$ is a very ample Fano polarization.

Proof. Let $L=\operatorname{Num}(X), s_{i j k} L \rightarrow L$ be the reflection considered in (6.7), $c, h$ the numerical classes of $C, H$; note that $s_{i j k}$ is an isometry of $L$ and that, by definition, $s_{i j k}(c)=h$. Therefore $H$ is constructed as in (1.2) from ten isotropic vectors satisfying (1.1). By (1.3) $|H|$ irreducible $\Rightarrow \phi_{H}$ is a birational morphism. Since $H$ has positive self-intersection the irreducibility of $|H|$ is equivalent to $h^{1}\left(\mathscr{O}_{X}(H)\right)=0$ [10]. Consider the exact sequence

$$
0 \rightarrow \mathscr{O}_{X}(H-C) \rightarrow \mathscr{O}_{X}(H) \rightarrow \mathscr{O}_{C}(H) \rightarrow 0 \text {. }
$$


Since $C \cdot(H-C)=1$ and $X_{C}$ does not contain lines, it follows that $h^{0}\left(\mathscr{O}_{X}(H-C)\right)=0$. Since $C \cdot(C-H)=-1$, it follows that

$$
h^{0}\left(\mathscr{O}_{X}\left(K_{X}+C-H\right)\right)=h^{2}\left(\mathscr{O}_{X}(H-C)\right)=0 .
$$

Then, by Riemann-Roch, $h^{1}\left(\mathscr{O}_{X}(H-C)\right)=0$. Furthermore $\mathscr{O}_{C}(H)$ is a degree 11 line bundle on a genus 6 curve so that $h^{1}\left(\mathscr{O}_{C}(H)\right)=0$. Hence, passing to the long exact sequence associated to (6.16), we obtain $h^{1}\left(\mathscr{O}_{X}(H)\right)=0$ and $|H|$ irreducible. By (1.3) $X_{H}$ has at most rational double points.

Let $l, m \in\{1 \cdots 10\}, l \neq m, F_{l m}=C-E_{l}-E_{m}$, and consider the exact sequence

$$
0 \rightarrow \mathscr{O}_{X}\left(F_{l m}-E_{n}\right) \rightarrow \mathscr{O}_{X}\left(F_{l m}\right) \rightarrow \mathscr{O}_{E_{n}}\left(F_{l m}\right) \rightarrow 0
$$

$(n \neq l, m)$. Observe that, with exactly the same proof used for $H-C$, $h^{0}\left(\mathscr{O}_{X}\left(F_{l m}-E_{n}\right)\right)=h^{1}\left(\mathscr{O}_{X}\left(F_{l m}-E_{n}\right)\right)=0$. Then, since $h^{0}\left(\mathscr{O}_{E_{n}}\left(F_{l m}\right)\right)=1$, it follows that $F_{l m}$ is an isolated curve in $X_{C}$. The degree of $F_{l m}$ is $4\left(C \cdot F_{l m}=\right.$ 4) and the arithmetic genus $1\left(F_{l m}^{2}=0\right)$. We can show that $F_{l m}$ is a nef divisor: since it is isolated $F_{l m}$ is not nef if and only if $D \cdot F_{l m}<0$ for some component $D \subset F_{l m}$. Now $F_{l m}$ is a quartic curve and $X_{C}$ does not contain lines; therefore either $F_{l m}$ is irreducible and nef because $F_{l m}^{2}=0$ or $F_{l m}=D_{1}+D_{2}$ with $D_{i}=$ smooth conic. In this latter case $D_{i}^{2}=-2$ so that $\left(D_{1}+D_{2}\right)^{2}=0 \Rightarrow D_{1} \cdot D_{2}=2 \Rightarrow D_{i} \cdot F_{l m}=0$; hence $F_{l m}$ is nef. Now assume $H$ is not very ample, then $X_{H}$ has a rational double point and $H \cdot R=0$ for some indecomposable nodal cycle $R$. Observe that

$$
H \sim F_{x y}+F_{z s}+E_{s}, \quad s \neq z,
$$

where $(x, y, z)$ is any permutation of $(i, j, k)$; then $R \cdot H=0 \Rightarrow R \cdot\left(F_{x y}+\right.$ $\left.F_{z s}+E_{s}\right)=0$ and, since $E_{s}$ is also nef, $F_{x y} \cdot R=F_{z s} \cdot R=E_{s} \cdot R=0$. In particular, $E_{s} \cdot R=0 \forall s=1 \cdots 10$ : on the other hand $C \sim \frac{1}{3} \sum E_{s}$ so that $C \cdot R=0$ : a contradiction because $C$ is very ample. Hence $H$ is very ample too.

Now let us fix

$$
\begin{aligned}
& X=\text { good nodal Enriques surface, } \\
& C=\text { good Reye polarization on } X, \\
& E_{i}, E_{i}^{\prime}=\text { the } 20 \text { plane cubics in } X_{C} .
\end{aligned}
$$

As above let $e_{i}=$ numerical class of $E_{i}, c=\frac{1}{3} \sum e_{i}=$ class of $C, r=$ $c-2 e_{1}=$ class of the rational quartic $R \subset X_{C}$ as in (6.6). Consider as in (6.13) the Fano vectors $c_{t}=\frac{1}{3} \sum e_{i}^{t}=h_{t}(c)$ and $f_{t}=e_{10}^{t}$, then

(6.20) Proposition. (i) $c_{t}$ is the numerical equivalence class of a very ample Fano polarization $C_{t}$;

(ii) $X_{C_{t}}$ does not contain lines.

Proof. By induction on $t(t=1)$. By definition

$$
h_{1}=f=s_{178} \cdot s_{256} \cdot s_{234} \text {. }
$$

Let $c=\frac{1}{3} \sum e_{i}$ be as above, we construct from $c$ the Fano vectors

$$
\begin{aligned}
a=s_{234}(c) & =\frac{1}{3} \sum e_{i}^{a}, \quad b=s_{256}(a)=\frac{1}{3} \sum e_{i}^{b}, \\
c_{1} & =s_{178}(b)=\frac{1}{3} \sum e_{i}^{1}=h_{1}(c) .
\end{aligned}
$$


Let $r=c-2 e_{1}$, and consider the intersection numbers

$$
x_{i}=r \cdot e_{i}, \quad a_{i}=r \cdot e_{i}^{a}, \quad b_{i}=r \cdot e_{i}^{b}, \quad y_{i}=r \cdot e_{i}^{1}
$$

and recall that $x_{1}=3, x_{i}=1, i \geq 2$ (cf. (6.13)). Then, applying the formulae (6.10) to $s_{234}, s_{256}, s_{178}$, it is easy to compute

$$
\begin{array}{ll}
\left(a_{1} \cdots a_{10}\right)=(3,2,2,2,1,1,1,1,1,1), & a \cdot r=5, \\
\left(b_{1} \cdots b_{10}\right)=(3,3,2,2,2,2,1,1,1,1), & b \cdot r=6, \\
\left(y_{1} \cdots y_{10}\right)=(4,3,2,2,2,2,2,2,1,1), & c_{1} \cdot r=7 .
\end{array}
$$

$a$ is the class of $A=2 C-E_{2}-E_{3}-E_{4}$. Since $C$ is good $A$ is very ample by Proposition (6.15). Let $L \subset X_{A}$ be a line, $l$ its numerical class. Since $X$ is good $l-r$ is divisible by 2 in $\operatorname{Num}(X)$; then $l \cdot e_{i}^{a}=a_{i} \bmod 2$ and, in particular, $l \cdot e_{i}^{a}$ is odd for $i \neq 2,3,4$. On the other hand $l \cdot e_{i}^{a} \geq 0$ because $e_{i}^{a}$ represents a nef divisor, (since $A$ is very ample $2 e_{i}^{a}$ corresponds to an elliptic pencil, cf. (1.4)). Then we compute $2<\frac{1}{3} \sum e_{i}^{a} \cdot l=a \cdot l=1$. Hence $X_{A}$ cannot contain lines. Now consider $b: b$ is the class of $B=2 A-E_{2}^{a}-E_{5}^{a}-E_{6}^{a}$ which is again a very ample Fano polarization by Proposition (6.15). Applying exactly the same arguments used for $X_{A}$ one shows that $X_{B}$ does not contain lines. So, by (6.15) again, $c_{1}$ is the class of the very ample Fano polarization $C_{1}=2 B-E_{1}^{b}-E_{7}^{b}-E_{8}^{b}$.

To complete the first step of induction we must show that $X_{C_{1}}$ does not contain lines: assume $L$ is a line (of numerical class $l$ ) in $X_{C_{1}}$, let $l_{i}=e_{i}^{1} \cdot l$. Since $X$ is good $l_{i}=y_{i} \bmod 2$. Then, using $(6.21)$ and $l \cdot c_{1}=1$, one obtains

$$
\left(l_{1} \cdots l_{10}\right)=(0,1,0,0,0,0,0,0,1,1) .
$$

Note that $s_{178}^{2}=$ id so that $s_{178}\left(e_{i}^{1}\right)=e_{i}^{b}$. Let $l_{i}^{\prime}-e_{i}^{b} \cdot l$; then, applying formulae (6.10), it is not difficult to compute $\left(l_{1}^{\prime} \cdots l_{10}^{\prime}\right)=(1,1,0,0,0,0,1,1,1,1)$ so that $b \cdot l=3$ : since $b \cdot r=6$ and $X$ is good this is a contradiction. Hence there is no line in $X_{C_{1}}$.

$(t>1)$ This time we start with $c_{t}=\frac{1}{3} \sum e_{i}^{t}$ and we assume $c_{t}=$ class of $C_{t}$, where $X_{C_{t}}$ is a smooth Fano model not containing lines. Recall that $c_{t+1}=h_{t}(c)=g\left(c_{t}\right)$ with $g=s_{178} \cdot s_{256} \cdot s_{243} \cdot s_{1910}$ (cf. (6.13)). Then consider the Fano vectors:

$$
\begin{array}{ll}
d=\frac{1}{3} \sum e_{i}^{d}=s_{1910}\left(c_{t}\right), & m=\frac{1}{3} \sum e_{i}^{m}=s_{234}(d), \\
n=\frac{1}{3} \sum e_{i}^{n}=s_{256}(m), & c_{t+1}=\frac{1}{3} \sum e_{i}^{t+1}=s_{178}(n),
\end{array}
$$

and the intersection numbers

$$
u_{i}=e_{i}^{t} \cdot r, \quad d_{i}=e_{i}^{d} \cdot r, \quad m_{i}=e_{i}^{m} \cdot r, \quad n_{i}=e_{i}^{n} \cdot r, \quad v_{i}=e_{i}^{t+1} \cdot r
$$

( $r$ as above). From (6.14) we know that

$$
u_{1}=2 t+2, \quad u_{2}=2 t+1, \quad u_{3}=\cdots=u_{8}=t+1, \quad u_{9}=u_{10}=t
$$

and that $c_{t} \cdot r=4 t+3$. Therefore, using the formulae (6.10), we can explicitly 
compute

$$
\begin{aligned}
\left(d_{1} \cdots d_{10}\right) & =(2 t+3,2 t+1, t+1, \ldots, t+1), \\
\left(m_{1} \cdots m_{10}\right) & =(2 t+3,2 t+2, t+2, t+2, t+1, \ldots, t+1), \\
\left(n_{1} \cdots n_{10}\right)= & (2 t+3,2 t+3, t+2, t+2, \\
t & +2, t+2, t+1, \ldots, t+1), \\
\left(v_{1} \cdots v_{10}\right)= & (2 t+4,2 t+3, t+2, \ldots, t+2, t+1, t+1) .
\end{aligned}
$$

Now the proof goes as in the case $t=1$ : let $L$ be any element of $\mathscr{R}(X)$, $l$ its class in $\operatorname{Num}(X)$. By (6.15) $D=2 C_{t}-E_{1}^{t}-E_{9}^{t}-E_{10}^{t}$ is a very ample Fano polarization of class $d$. Let $l_{i}^{d}=l \cdot e_{i}^{d}$, then $l_{i}^{d} \geq 0$ because $e_{i}^{d}$ represents a nef divisor. Since $X$ is good one computes from (6.22): $t$ even $\Rightarrow\left(l_{1}^{d}, \ldots, l_{10}^{d}\right)=(1, \ldots, 1) \bmod 2 \Rightarrow l \cdot d \geq 3 ; t$ odd $\Rightarrow\left(l_{1}^{d}, \ldots, l_{10}^{d}\right)=$ $(1,1,0, \ldots, 0) \bmod 2 \Rightarrow l \cdot d \geq 2$. This implies that there is no line in $X_{D}$ and that $M=2 D-E_{2}^{d}-E_{3}^{d}-E_{4}^{d}$ is a very ample Fano polarization representing $m$. Now we just go on in the same way: let $l_{i}^{m}=l \cdot e_{i}^{m}$; for the same reasons as above we have $l_{i}^{m} \geq 0$ and

$$
\begin{aligned}
& \left(l_{1}^{m} \cdots l_{10}^{m}\right)=(1,0,0,0,1,1,1,1,1,1) \bmod 2, \quad(t \text { even }), \\
& \left(l_{1}^{m} \cdots l_{10}^{m}\right)=(1,0,1,1,0,0,0,0,0,0) \bmod 2, \quad(t \text { odd }) .
\end{aligned}
$$

This implies that $l \cdot m=1$ if and only if $t$ is odd and

$$
\left(l_{1}^{m} \cdots l_{10}^{m}\right)=(1,0,1,1,0,0,0,0,0,0) .
$$

But now, if such an $l$ exists, we have $l \cdot s_{234}\left(e_{2}^{m}\right)=l \cdot\left(m-e_{3}^{m}-e_{4}^{m}\right)=-1$; impossible because $s_{234}\left(e_{2}^{m}\right)=e_{2}^{d}=$ class of plane cubic in $X_{D}=$ class of a nef divisor. Therefore $N=2 M-E_{2}^{m}-E_{5}^{m}-E_{6}^{m}$ is a very ample Fano polarization representing $n$. Let $l_{i}^{n}=l \cdot e_{i}^{n}$ then $l_{i}^{n} \geq 0$ and we have this time

$$
\begin{aligned}
& \left(l_{1}^{n} \cdots l_{10}^{n}\right)=(1,1,0,0,0,0,1,1,1,1) \bmod 2, \quad(t \text { even }), \\
& \left(l_{1}^{n} \cdots l_{10}^{n}\right)=(1,1,1,1,1,1,0,0,0,0) \bmod 2, \quad(t \text { odd }) .
\end{aligned}
$$

Clearly $l \cdot n \geq 2$ so that $X_{N}$ does not contain lines. Finally we obtain from this the very ample Fano polarization $C_{t+1}=2 N-E_{1}^{n}-E_{7}^{n}-E_{8}^{n}$ of class $c_{t+1}$. Let $l_{i}=c_{t+1} \cdot l$, then

$$
\begin{aligned}
& \left(l_{1} \cdots l_{10}\right)=(0,1,0,0,0,0,0,0,1,1) \bmod 2, \quad(t \text { even }), \\
& \left(l_{1} \cdots l_{10}\right)=(1,1,1,1,1,1,0,0,0,0) \bmod 2, \quad(t \text { odd }) .
\end{aligned}
$$

If $t$ is odd $l \cdot c_{t+1} \geq 2$ and $X_{C_{t+1}}$ does not contain lines; if $t$ is even it is completely clear how to complete the proof using the previous arguments.

Finally we can show

(6.22) Theorem. Let $X$ be a good nodal Enriques surface, $m: \mathscr{E}(X) \times \mathscr{R}(X) \rightarrow$ $\mathbf{N}$ the intersection map considered in (6.1). Then

(1) $m$ is surjective;

(2) $X$ admits a Reye polarization of index $t$ for each $t \geq 3$.

Proof. Fix on $X$ a good Reye polarization $C$ and its numerical class $c=$ $\frac{1}{3} \sum e_{i}$. Then, as in (6.13), reconstruct from $c$ the Fano vectors

$$
c_{t}=\frac{1}{3} \sum e_{i}^{t} \quad(t \geq 1)
$$


and consider also $r=c-2 e_{1}, f_{t}=e_{10}^{t}$. By (6.6) $r$ is represented by an element of $\mathscr{R}(X)$ (a rational quartic curve $R$ in $X_{C}$ ). By $(6.14) r \cdot f_{t}=t$. By Proposition $(6.20) c_{t}$ is the class of a very ample Fano polarization $C_{t}$. Hence, by (1.4), $f_{t}$ is represented by $F_{t}=$ plane cubic curve in $X_{C_{t}}=$ isolated elliptic curve on $X$. Therefore $F_{t} \in \mathscr{E}(X)$ and $m\left(F_{t}, R\right)=t, t \geq 1$. On the other hand, it is a standard fact that for a given $R \in \mathscr{R}(X)$ there exists $E \in \mathscr{E}(X)$ such that $E \cdot R=0$. Therefore $m$ is surjective. Finally, by (4.12), the surjectivity of $m$ implies (2).

(6.23) Remark (Cremona transformations of $\mathbf{P}^{5}$ ). We want to explain without proofs the true geometric construction underlying the numerical arguments used in the section. Fix three distinct planes $\pi_{i}, \pi_{j}, \pi_{k}$ in $\mathbf{P}^{5}$ such that: (i) there is no hyperplane containing all of them, (ii) any two of them intersect exactly in one point. Then consider the linear system $\Sigma$ of the quadrics containing $\pi_{i} \cup \pi_{j} \cup \pi_{k}: \quad \Sigma$ is 5 -dimensional and defines a birational transformation $s_{i j k}: \mathbf{P}^{5} \rightarrow \mathbf{P}^{5}$, this is called a standard Cremona transformation of $\mathbf{P}^{5}$. Under $s_{i j k}$ each $\pi_{m}(m=i, j, k)$ is blown up to a hyperplane, while the hyperplanes containing any two of $\pi_{i}, \pi_{j}, \pi_{k}$ are contracted to three new planes satisfying (i), (ii). Taking them and constructing the corresponding Cremona transformation we obtain the inverse of $s_{i j k}$. Let $X_{C} \subset \mathbf{P}^{5}$ be a smooth Fano model, $X_{C}$ not contained in a quadric, (note that this is always possible up to replacing $C$ by $\left.C+K_{X}\right)$. Assume that three plane cubics of $X_{C}$, e.g. $E_{i}, E_{j}, E_{k}$, are contained in $\pi_{i}, \pi_{j}, \pi_{k}$; then $s_{i j k} / X_{C}$ is the morphism associated to $\left|2 C-E_{i}-E_{j}-E_{k}\right|$ and, at least if there is no line in $X_{C}, s_{i j k}\left(X_{C}\right)$ is a new Fano model by (6.18). Therefore, thinking more geometrically, the reflections $s_{i j k}: \operatorname{Num}(X) \rightarrow \operatorname{Num}(X)$ we have used throughout this section could be considered as Cremona transformations of $\mathbf{P}^{5}$ applied to a suitable Fano model.

\section{REFERENCES}

1. E. Arbarello, M. Cornalba, P. Griffiths, and J. Harris, Geometry of algebraic curves. I, Springer-Verlag, Berlin, 1984.

2. I. Shafarevich, Algebraic surfaces, Proc. Steklov Inst. Math. 75 (1964).

3. W. Barth, Lectures on K3 and Enriques surfaces, Algebraic Geometry, Sitges 1983, Lecture Notes in Math., vol. 1124, Springer, Berlin, 1985, pp. 21-57.

4. W. Barth, C. Peters, and A. Van de Ven, Complex algebraic surfaces, Springer-Verlag, Berlin, 1984.

5. A. Beauville, Surfaces algébriques complexes, Astérisque 54, Soc. Mat. de France, 1980.

6. A. Coble, The ten nodes of the rational sextic and of the Cayley symmetroid, Amer. J. Math. 40 (1918), 317-340.

7. F. Cossec, On the Picard group of Enriques surfaces, Math. Ann. 271 (1985), 577-600.

8. __ Reye congruences, Trans. Amer. Math. Soc. 280 (1983), 737-751.

9. F. Cossec and I. Dolgachev, Rational curves on Enriques surfaces, Math. Ann. 272 (1985), 369-384.

10. __ Enriques surfaces. I, Birkhaüser, Basel, 1989; II (to appear).

11. I. Dolgachev and I. Reider, On rank 2 vector bundles with $c_{1}^{2}=10$ and $c_{2}=3$ on Enriques surfaces, Proc. USA-URSS Conf. on Algebraic Geometry, Chicago, 1989.

12. P. Le Barz, Formules multisecantes pour les courbes gauches quelconques, Enumerative Geometry and Classical Algebraic Geometry, Birkhäuser, Basel, 1982, pp. 165-198. 
13. E. L. Livorni, On the existence of some surfaces, Algebraic Geometry: Proc. Internat. Conf. (L'Aquila, 1988), Lecture Notes in Math., vol. 1417, Springer, Berlin, 1990.

14. A. Verra, On Enriques surfaces as a fourfold cover of $\mathbf{P}^{2}$, Math. Ann. 266 (1983), 241-250.

15. Hoil Kim, Stable vector bundles on Enriques surfaces, Ph.D. thesis, Univ. of Michigan, 1990.

Dipartimento di Matematica, Università di Torino, via C. Alberto 10, 10123 Torino, ITALY

Dipartimento di Matematica, Università di Genova, via L. B. Alberti 4, 16132 Genova, ITALY 